\title{
Ensheathing Glia Function as Phagocytes in the Adult Drosophila Brain
}

\author{
Johnna Doherty, ${ }^{*}$ Mary A. Logan, ${ }^{*}$ Özge E. Taşdemir, and Marc R. Freeman \\ Department of Neurobiology, University of Massachusetts Medical School, Worcester, Massachusetts 01605-2324
}

The mammalian brain contains many subtypes of glia that vary in their morphologies, gene expression profiles, and functional roles; however, the functional diversity of glia in the adult Drosophila brain remains poorly defined. Here we define the diversity of glial subtypes that exist in the adult Drosophila brain, show they bear striking similarity to mammalian brain glia, and identify the major phagocytic cell type responsible for engulfing degenerating axons after acute axotomy. We find that neuropil regions contain two different populations of glia: ensheathing glia and astrocytes. Ensheathing glia enwrap major structures in the adult brain, but are not closely associated with synapses. Interestingly, we find these glia uniquely express key components of the glial phagocytic machinery (e.g., the engulfment receptor Draper, and dCed-6), respond morphologically to axon injury, and autonomously require components of the Draper signaling pathway for successful clearance of degenerating axons from the injured brain. Astrocytic glia, in contrast, do not express Draper or dCed-6, fail to respond morphologically to axon injury, and appear to play no role in clearance of degenerating axons from the brain. However, astrocytic glia are closely associated with synaptic regions in neuropil, and express excitatory amino acid transporters, which are presumably required for the clearance of excess neurotransmitters at the synaptic cleft. Together these results argue that ensheathing glia and astrocytes are preprogrammed cell types in the adult Drosophila brain, with ensheathing glia acting as phagocytes after axotomy, and astrocytes potentially modulating synapse formation and signaling.

\section{Introduction}

Glia are the most abundant cell type in the mammalian nervous system, accounting for $\sim 90 \%$ of cells in the mature brain. The different subtypes of mammalian glia-astrocytes, oligodendrocytes, microglia, and Schwann cells- have been extensively classified based on morphology, molecular markers, and position within the nervous system and are thought to play largely distinct roles in nervous system development and function. In the CNS, astrocytes provide trophic support for neuronal growth and regulate synapse formation and signaling. Oligodendrocytes ensheath and myelinate axons, thereby protecting axons and providing a stable ionic environment for proper conduction of action potentials. Microglia act as the resident immune cells in the brain, responding to infection and neural trauma, acting as phagocytes, and mediating posttrauma events (for review, see Barres, 2008).

The developing Drosophila embryonic and larval nervous sys-

\footnotetext{
Received Dec. 15, 2008; revised March 5, 2009; accepted March 9, 2009.

This work was supported by National Institutes of Health Grant 1R01NS053538. M.A.L. is supported by an American Cancer Society Postdoctoral Fellowship (Grant PF-07-258-01-CSM). M.R.F. is an Alfred Sloan Research Fellow and was supported by a Smith Family New Investigator Award from the Smith Family Foundation (Chesnut Hill, MA). We thank T. Awasaki, M. Bhat, S. Birman, B. Dickson, T. Lee, Y. Nakanishi, S. Waddell, C. Warr, Bloomington Stock Center, and Developmental Studies Hybridoma Bank for sharing fly stocks, antibodies, and plasmids. We thank T. Lee for the gift of the repo-Gal80 line. We thank Jennifer Ziegenfuss for help with the production and imaging of MARCM clones. We thank Takeshi Awasaki and Tzumin Lee for helpful discussions regarding the production of adult brain glial clones.

*J.D. and M.A.L. contributed equally to this work.

The authors declare no competing financial interests.

Correspondence should be addressed to Marc R. Freeman at the above address. E-mail:marc.freeman@umassmed.edu. DOI:10.1523/JNEUROSC1.5951-08.2009

Copyright $\odot 2009$ Society for Neuroscience $\quad 0270-6474 / 09 / 294768-14 \$ 15.00 / 0$
}

tems contain unique subsets of glial cells that are morphologically and molecularly similar to their mammalian counterparts (Ito et al., 1995; Edenfeld et al., 2005; Freeman and Doherty, 2006, Logan and Freeman, 2007). Drosophila glia play critical roles during the formation of the nervous system, including regulation of axon pathfinding (Hidalgo and Booth, 2000; Poeck et al., 2001), engulfment of apoptotic neurons (Sonnenfeld and Jacobs, 1995; Freeman et al., 2003), and nerve ensheathment (Auld et al., 1995; Leiserson et al., 2000; Silies et al., 2007). Much less is known about adult brain glia in Drosophila, although exciting recent evidence suggests that glia in the adult Drosophila brain play a central role in the function and health of the brain. For example, in the adult brain, Ebony, a $N$ - $\beta$-alanyl-biogenic amine synthetase, acts in glia to modulate circadian locomotor behavior (Suh and Jackson, 2007); glial cells also signal to one another in the adult through the 7-transmembrane G-protein-coupled receptor Moody to actively maintain a pleated septate junctionbased blood-brain barrier that isolates the CNS from nonneuronal tissues (Bainton et al., 2005; Schwabe et al., 2005); and glial cells act as phagocytes in the injured brain and engulf degenerating axonal debris after trauma (MacDonald et al., 2006). With the exception of blood-brain barrier formation, which appears to be mediated by a population termed "surface glia," the subtypes of glia that regulate the diversity of glial functions in the adult Drosophila brain remain to be identified.

Here we explore the diversity of glial cell populations found in the adult Drosophila CNS to determine whether unique subtypes of glia exist and how they might differ in morphology, location within the CNS, gene expression patterns, and function. Using the olfactory system as a model tissue, we identify three unique 


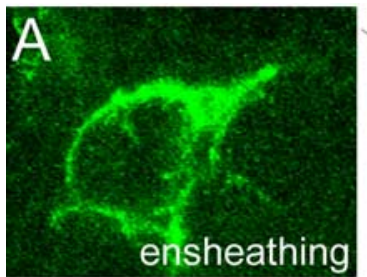

\section{glial nucleus \\ neuropil \\ neuronal cell body}

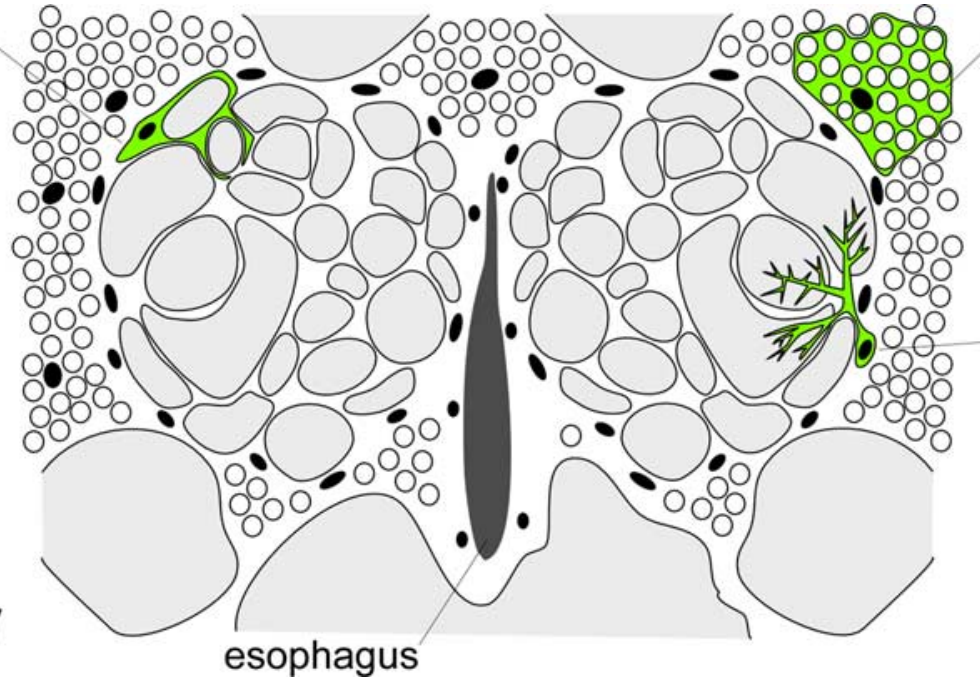

Figure 1. Identification of morphologically distinct subtypes of glial cells in the adult Drosophila brain. repo-Gal 4 was used to label MARCM glial clones with GFP, and the morphology of individual glial cells was analyzed in the adult antennal lobe brain region. We identified three major subtypes of glial cells: ensheathing glia, cortex (or cell body) glia, and astrocytes. Cartoon schematic of the adult antennal lobe brain region depicts the standard position of cell bodies and approximate sizes of each glial cell type within the brain. Confocal Z-stack projections of representative MARCM clones of each glial subtype are shown in $\boldsymbol{A}-\boldsymbol{C}$. $\boldsymbol{A}$, Ensheathing glia had a flattened appearance with relatively few branch points, and their membranes appeared to surround and demarcate distinct compartments of the neuropil. $\boldsymbol{B}$, Cortex glia resided outside the neuropil in the cortex where neuronal cell bodies are found, and appeared to fully ensheath the soma of every brain neuron within its spatial domain. C, Astrocytes projected into the neuropil a major stalk that branched and ramified profusely, ultimately positioning astrocyte membrane processes in close proximity to the synapse-rich regions of the glomeruli.

glial subtypes, differing in morphology, patterns of gene expression, and function. We identify "ensheathing glia" as the subtype that are molecularly and functionally competent to act as phagocytes after acute axotomy. We also identify an astrocytic subtype of glia in Drosophila, which does not appear to respond morphologically to axotomy or be required for clearance of degenerating axons. Thus, uniquely identifiable and functionally distinct subclasses of glia exist in the mature Drosophila brain, and these bear striking molecular and functional similarity to the major glial subtypes found in the mammalian brain.

\section{Materials and Methods}

Fly strains. The following Drosophila strains were used: repo-Gal4 (Leiserson et al., 2000), mZ0709-Gal4 (Ito et al., 1995), repo-Gal80 (a kind gift from T. Lee, University of Massachusetts Medical School, Worcester, MA), pUAST- $m$ CD8::GFP (Lee and Luo, 2001), OR85e-mCD8::GFP (gift from B. Dickson, Research Institute of Molecular Pathology, Vienna, Austria), pUAST-ced-6-RNAi (Awasaki et al., 2006), pUAST-shark-RNAi (Ziegenfuss et al., 2008), pUAST-draper-RNAi (MacDonald et al., 2006), draper ${ }^{\Delta 5}$ (MacDonald et al., 2006), $D f(2 R) w 73-1, \mathrm{cn}^{1} / C y O$ (Bloomington Drosophila Stock Center), UAS-shibire ${ }^{t s}, y w, h s-F L P ; F R T G 13, T u b-G a l 80$ (a kind gift from T. Lee), FRTG13;UAS-mCD8::GFP, repo-Gal4, FRTG13,UAS-mCD8::GFP; alrm-Gal4, FRTG13,UAS-mCD8::GFP;mz0709-Gal4, eaat1-Gal4 (Rival et al., 2004), and $w$,hs-flp;FRT82B,UAS-mCD8::GFP (a kind gift from T. Lee) and w;eaat1-gal4;FRT82B,Tub-Gal80.

The alrm-Gal4 construct was generated by amplifying a 4973 bp region of the CG11910 (named astrocytic leucine-rich repeat molecule, alrm) promoter using the following primers: $5^{\prime}$-GATCGATCGCGGCCGCTAGTGGCGATCCTTTCGCTCG-3' and 5'-GATCGGTACCGAGTTAATATGGTGGGAACTGC- $3^{\prime}$. The resulting fragment was then cloned into the PG4PN2 vector (a gift from C. Warr, Monash University, Victoria, Australia), and transgenic flies were generated using standard methods by BestGene.

Olfactory receptor neuron injury protocol. Maxillary palp and antennal ablations were performed as previously described (MacDonald et al., 2006). A detailed description of quantitative analysis of Draper recruitment and clearance of severed axons is provided in the supplemental material (supplemental Methods and supplemental Fig. 3, available at www.jneurosci.org).
Shibire $e^{\text {ts }}$ experiments. UAS-shibire ${ }^{\text {ts }}$ flies were crossed to the appropriate Gal 4 driver lines. Flies were raised at $18^{\circ} \mathrm{C}$, shifted to $30^{\circ} \mathrm{C} 2 \mathrm{~d}$ before maxillary palp ablation, and maintained at $30^{\circ} \mathrm{C}$ until ready for immunohistochemistry.

Immunohistochemistry and confocal microscopy. Adult brains were dissected, fixed, and antibody stained using standard techniques (MacDonald et al., 2006). Confocal imaging and quantitation of Draper staining and green fluorescent protein (GFP) were performed as previously described (MacDonald et al., 2006). The following primary antibodies were used: 1:200 mouse anti-GFP (Invitrogen), 1:500 rabbit anti-GFP (Invitrogen), 1:500 rabbit anti-Draper (Freeman et al., 2003), 1:5 mouse anti-Repo (Developmental Studies Hybridoma Bank), 1:1000 guinea pig anti-Repo (a gift from M. Bhat, University of North Carolina, Chapel Hill, NC), 1:20 mouse anti-nc82 (Developmental Studies Hybridoma Bank), and 1:500 rat anti-dCed-6 (Awasaki et al., 2006). All anti-IgG secondary antibodies were FITC, Cy3, or Cy5 conjugated (Jackson ImmunoResearch) and used at 1:200.

Mosaic analysis with repressible cell marker clone production. Glial mosaic analysis with repressible cell marker (MARCM) clones were made by heat shocking 36 - to 72 -h-old larvae in a $37^{\circ} \mathrm{C}$ water bath for $30 \mathrm{~min}$. Flies were then maintained at $25^{\circ} \mathrm{C}$ until anti-GFP immunohistochemistry was performed.

\section{Results}

In an effort to identify distinct morphological subtypes of glial cells in the mature Drosophila brain, we used the MARCM system (Lee and Luo, 2001) to generate small clones of glial cells labeled with membrane-tethered GFP. Larvae containing a hs-flipase allele and a wild-type chromosome arm for recombination were subjected to a short heat shock $\left(37^{\circ} \mathrm{C}\right)$ and glial cells within clones were visualized by use of the pan-glial driver, repo-Gal4. Our analysis of glial subtype morphology is focused mainly on the adult antennal lobe region (cartoon schematic depicted in Fig. 1) owing to its well defined histology and accessibility to genetic manipulation. In the adult brain we identified clones resembling each of the three main types of glial cells found in embryos and larvae: (1) cortex glia, which resided outside the neuropil in regions housing neuronal cell bodies, ramified dramatically to sur- 


\section{control}
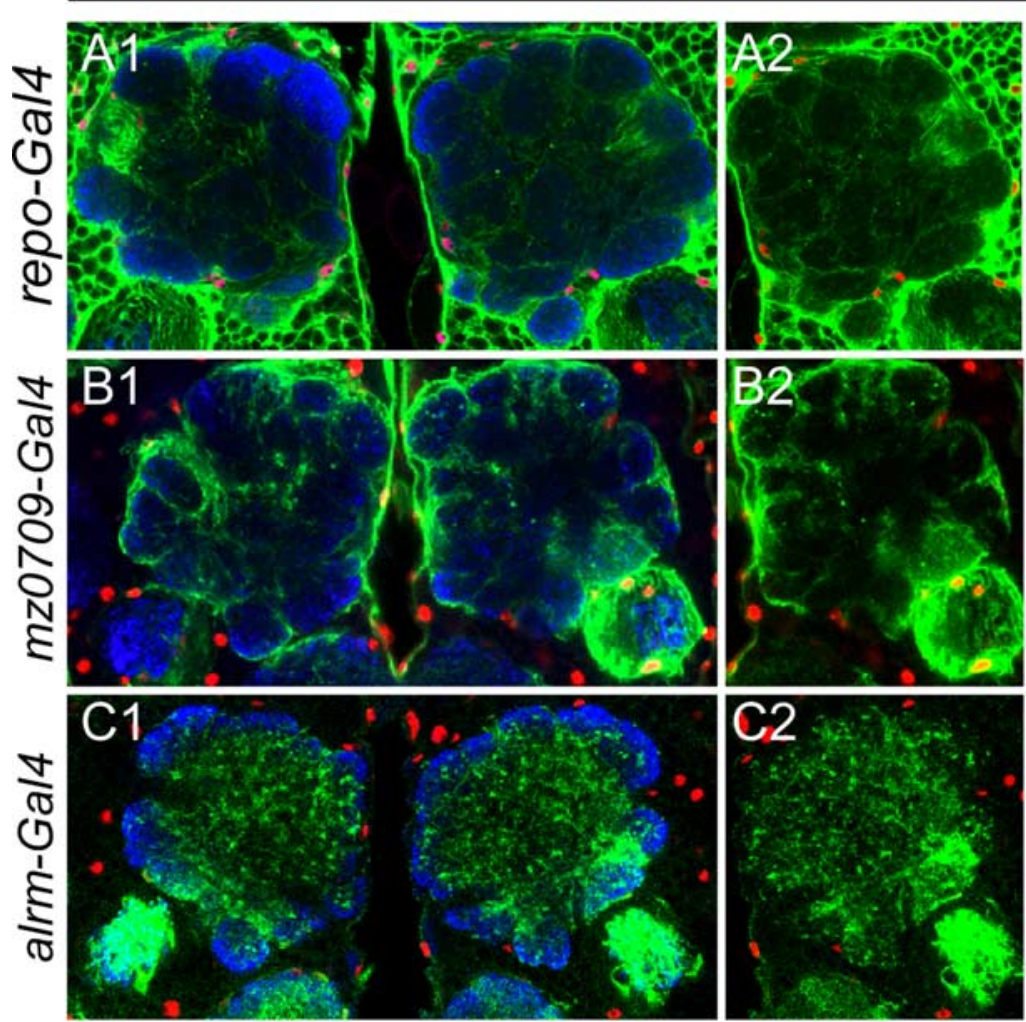
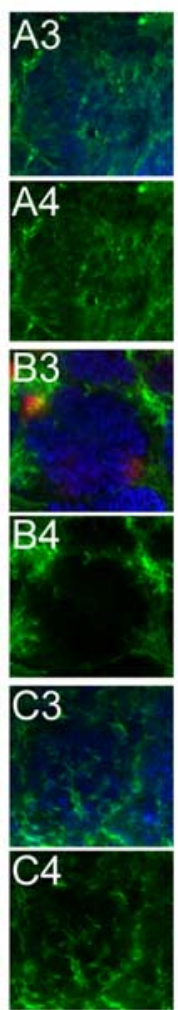

repo-Gal80

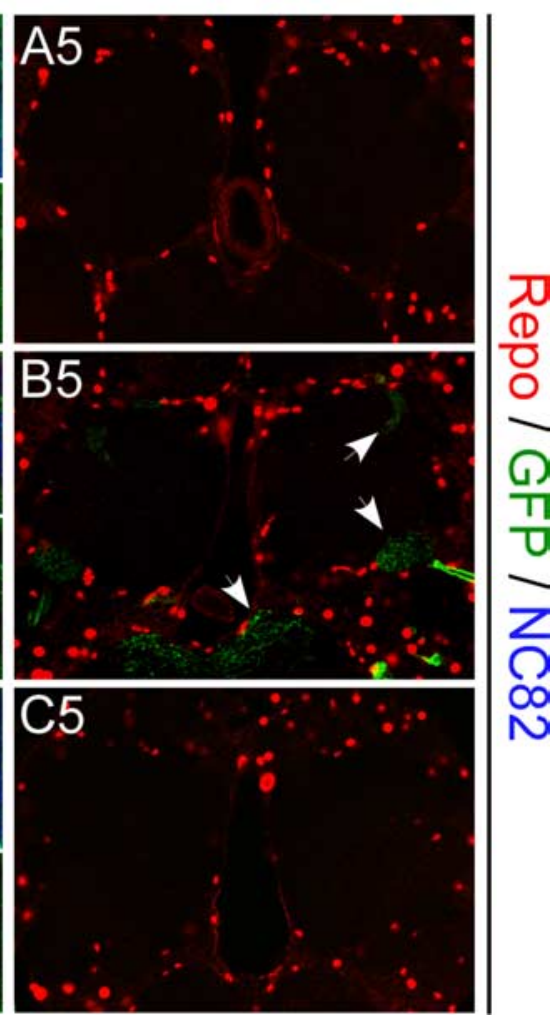

Figure 2. Characterization of Gal4 drivers that uniquely label astrocytes and ensheathing glial subtypes. Individual Gal4 drivers were crossed to UAS- $m$ CD8::GFP, and stained for GFP ( $\alpha$-GFP, green), neuropil (nc82, blue), and glial nuclei ( $\alpha$-Repo, red). Panels show single confocal slices of both antennal lobes $(\boldsymbol{A 1}, \boldsymbol{A 5}, \boldsymbol{B 1}, \boldsymbol{B 5}, \boldsymbol{C 1}, \mathbf{C 5})$, a single antennal lobe without nc82 stain $(\boldsymbol{A 2}, \boldsymbol{B 2}$, C2), or a high-magnification view of a single glomerulus (top, with nc82; bottom, without) $(\boldsymbol{A} \mathbf{3}, \boldsymbol{A 4}, \boldsymbol{B} \mathbf{3}, \boldsymbol{B} 4, \mathbf{C}, \mathbf{C} 4)$. A minimum of 10 animals were imaged for each experiment with similar patterns of expression observed. $\boldsymbol{A}$, repo-Gal4-driven mCD8::GFP labeled all glial membranes (A1, $\boldsymbol{A 2}$ ), which were seen surrounding and invading glomeruli (A2-A4). All mCD8::GFP expression was suppressed in this genetic background by repo-Gal80 (A5). $\boldsymbol{B}$, mz0709-Gal4 labeled ensheathing glia that surround glomeruli (B1, $\boldsymbol{B 2}$ ), but their membranes did not invade glomeruli (B2-B4). Nearly all GFP expression was suppressed by repo-Gal80 (B5), indicating that this driver is largely specific to glia. However, in some brains, a small number of neurons remained GFP labeled (arrowheads). These cells were identified as neurons by tracing axons to Repo ${ }^{-}$neuronal cell bodies. C, alrm-Gal4 labeled only astrocytes (C1, (2), which projected ramified processes that deeply invaded glomeruli (C2-C4), and all GFP expression driven by this Gal4 line was suppressed by repo-Gal80 (C5).

round individual cell bodies (Fig. $1 B$ ); (2) surface glia, which appeared as large flat cells enveloping the surface of the brain, did not extend any processes into the brain (data not shown); and (3) neuropil glia, which were closely associated with the neuropil, extended membranes into synaptic regions, and surrounded large bundles of axons (Fig. $1 A, C$ ). As in the embryo and larva, glial cell bodies were not found within the neuropil, rather they resided at the edge of the neuropil (neuropil glia), in the cortex (cortex glia), or at the surface of the brain (surface glia).

Interestingly, the single-cell resolution provided by MARCM analysis allowed us to further subdivide neuropil glia into two distinct morphological classes, "ensheathing glia" and "astrocytic glia.” Ensheathing glia (Fig. $1 A$ ) appeared as flattened cells that lined the borders of the neuropil and subdivided regions of the brain by isolating neuropil from the surrounding cortex. Within the antennal lobe, ensheathing glial membranes surrounded individual glomeruli (the functional units of the antennal lobe) but did not extend into the synaptic regions of the glomeruli. In addition, we identified an astrocyte-like cell type (Fig. 1C) that extended membrane processes deeply into the neuropil and ramified profusely in synaptic-rich regions. This latter cell type we refer to as the fly "astrocyte," based on its striking morphological similarity to mammalian astrocytes, as well as the conserved expression of a number of molecular markers used to identify as- trocytes in the mammalian brain. Mammalian astrocytes remove excess amounts of extracellular glutamate through the highaffinity excitatory amino acid transporters (EAATs), GLAST and GLT-1, which transports the glutamate into glial cells where it is then converted into glutamine by glutamine synthetase (Chaudhry et al., 1995; Lehre et al., 1995; Rival et al., 2004). We found that Drosophila astrocytes also express the transporter EAAT1 (supplemental Fig. 1, available at www.jneurosci.org as supplemental material). While we used the adult antennal lobe as our primary model tissue in this study, we observed the morphological glial subtypes described above in all brain regions examined, suggesting that our results are generally applicable to glial populations throughout the adult Drosophila brain (supplemental Fig. 2, available at www.jneurosci.org as supplemental material).

We next sought to identify Gal4 driver lines that would allow us to uniquely label and manipulate these glial populations, with our major focus being to genetically subdivide neuropil glia (i.e., ensheathing glia versus astrocytes). To accomplish this, we crossed UAS-mCD8::GFP to a previously described collection of embryonic and larval glial drivers (Ito et al., 1995), as well as a number of drivers generated in our own laboratory. We then looked in the adult antennal lobe to examine the morphology and spatial distribution of cell types marked by these drivers in a 
control
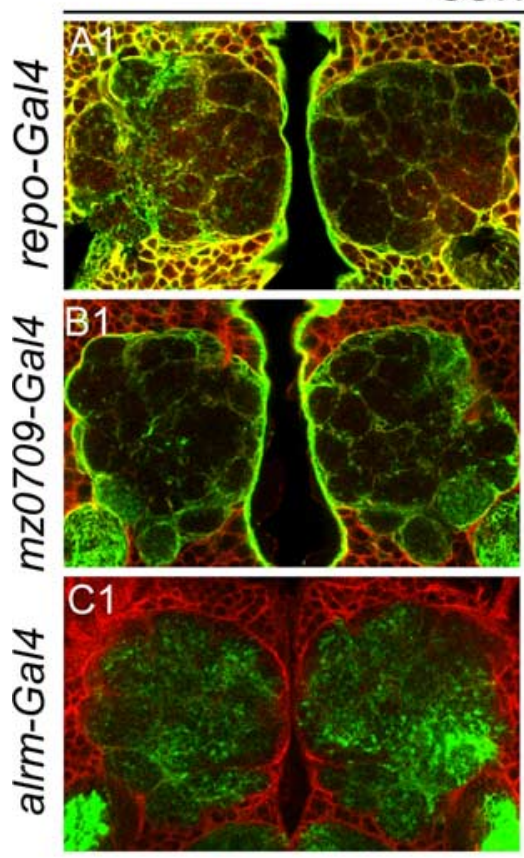
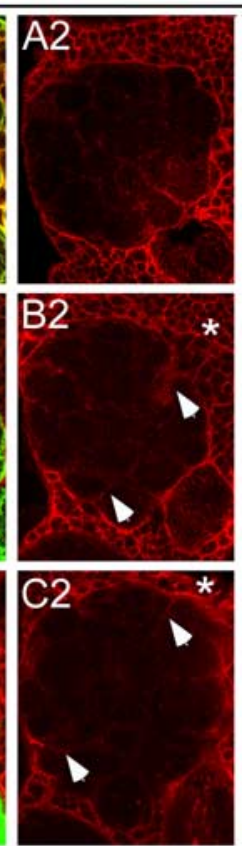

draper $r^{R N A i}$
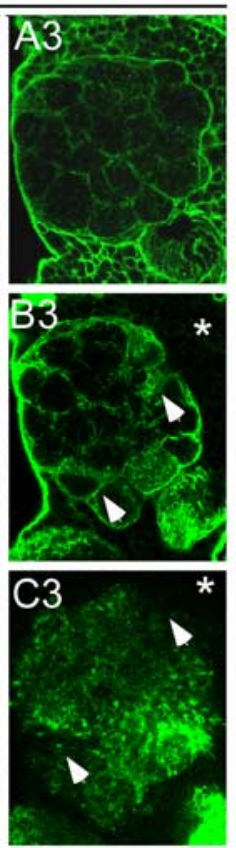
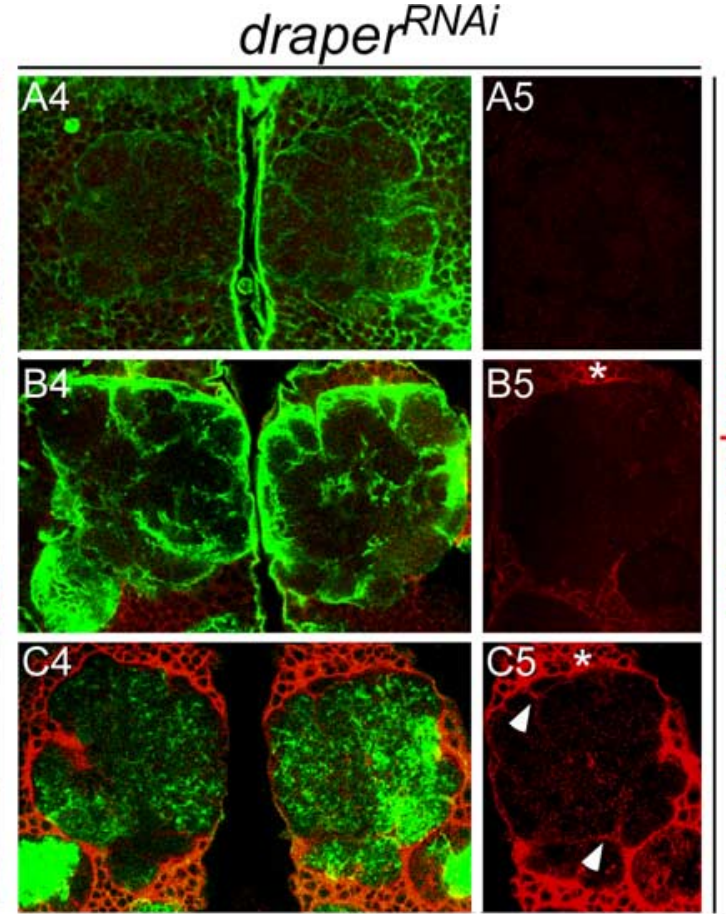

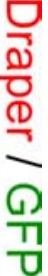

\section{high magnification of glomeruli in antennal lobe}

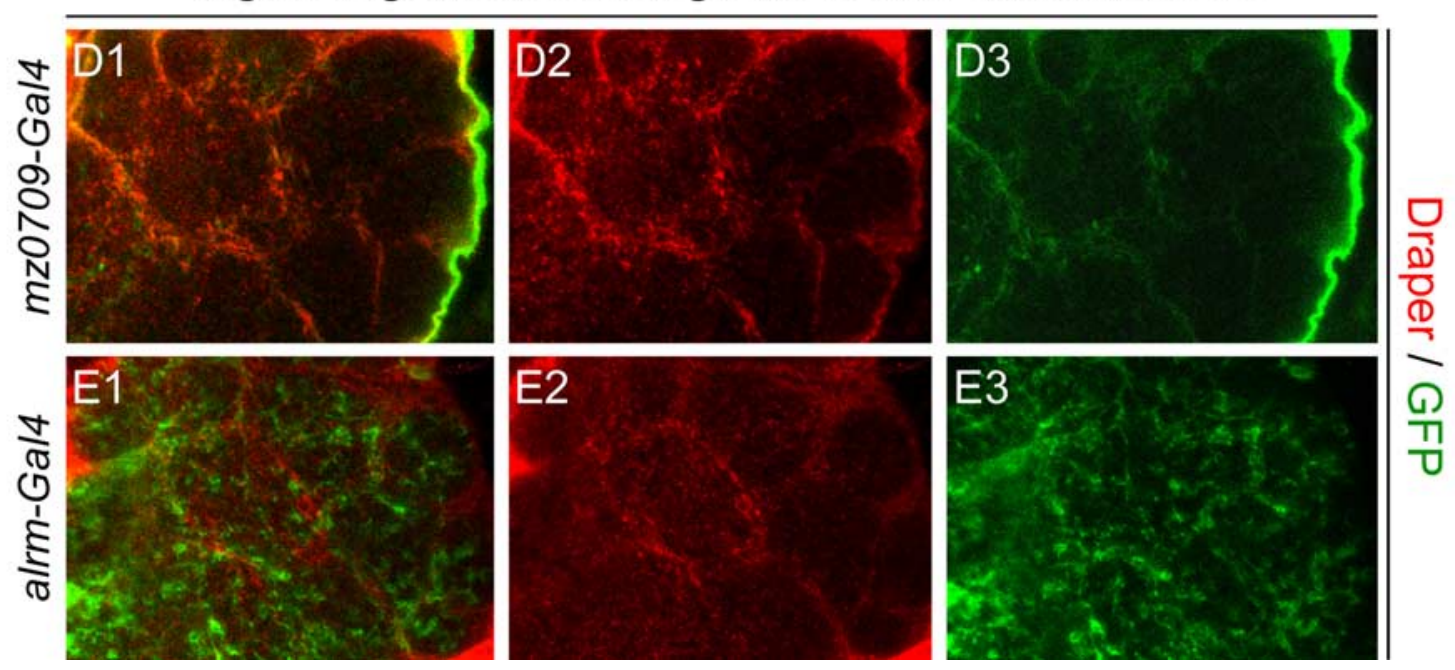

Figure 3. The engulfment receptor Draper is expressed in ensheathing and cortex glia but not in astrocytes. Flies carrying UAS-mCD8::GFP were crossed to each glial subtype driver line. Glial

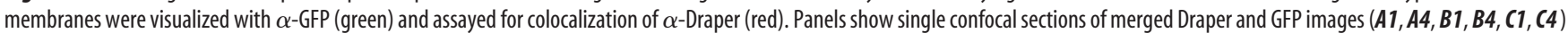
as well as single antennal lobes showing either GFP or Draper staining alone $(\boldsymbol{A 2}, \boldsymbol{A 3}, \boldsymbol{A 5}, \boldsymbol{B} \mathbf{2}, \boldsymbol{B} \mathbf{3}, \boldsymbol{B} \mathbf{5}, \mathbf{C} \mathbf{2}, \mathbf{C} \mathbf{3}, \mathbf{C 5})$. A minimum of 10 animals were imaged for each genotype with similar results. $\boldsymbol{A}$, Pan-glial expression of mCD8::GFP with repo-Gal4 resulted in extensive colocalization of Draper and GFP-labeled membranes in the neuropil and cortex of the brain (A1-A3). No Draper immunoreactivity was detectable when UAS-draper ${ }^{R N A i}$ was expressed in the same genetic background $(\boldsymbol{A 4}, \boldsymbol{A 5})$. $\boldsymbol{B}$, Driving mCD8::GFP with $m z 0709-G a l 4$ resulted in extensive colocalization of Draper and GFP-labeled membranes (arrowheads) at the edges of the neuropil and surrounding individual glomeruli, but not in the cortex (asterisk; B1-B3). When mzO709-Gal4 was used to drive UASdraper $^{R N A i}$, Draper immunoreactivity was absent immediately surrounding and within the neuropil but was still detectable in the cell cortex (B4, $\boldsymbol{B 5}$; asterisk). $\boldsymbol{C}$, Labeling of astrocyte membranes with alrm-Gal4-driven mCD8::GFP resulted in no detectable colocalization of Draper and GFP (C1-C3; arrowheads, asterisk), and expression of UAS-draper ${ }^{R N A i}$ in astrocytes had no effect on Draper levels in the cortex or neuropil (C4, C5; arrowheads, asterisk). D, High-magnification view of glomeruli within the antennal lobe shows Draper and mz0709-Gal4-driven GFP colocalizing on

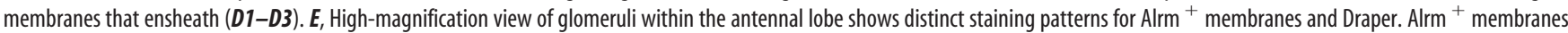
innervate but do not wrap around glomeruli.

background with glial nuclei ( $\alpha$-Repo) and the neuropil ( $\alpha$-nc82) also labeled. The repo-Gal4 driver labeled all Repo ${ }^{+}$glial subtypes in the adult brain, as evidence by $\alpha$-Repo immunostaining in the nuclei of $\mathrm{GFP}^{+}$cells (Fig. $2 A$ ). Membrane processes from Repo cells are found throughout the adult brain, and together they constitute the diverse collection of glial subtypes identified in our single-cell MARCM analysis. Upon examination of a single glomerulus within the antennal lobe, we found that membranes from Repo ${ }^{+}$cells both surround and invade glomeruli (Fig. $2 A 3, A 4)$. All GFP expression in the adult brain driven by the 


\section{1 day after injury}
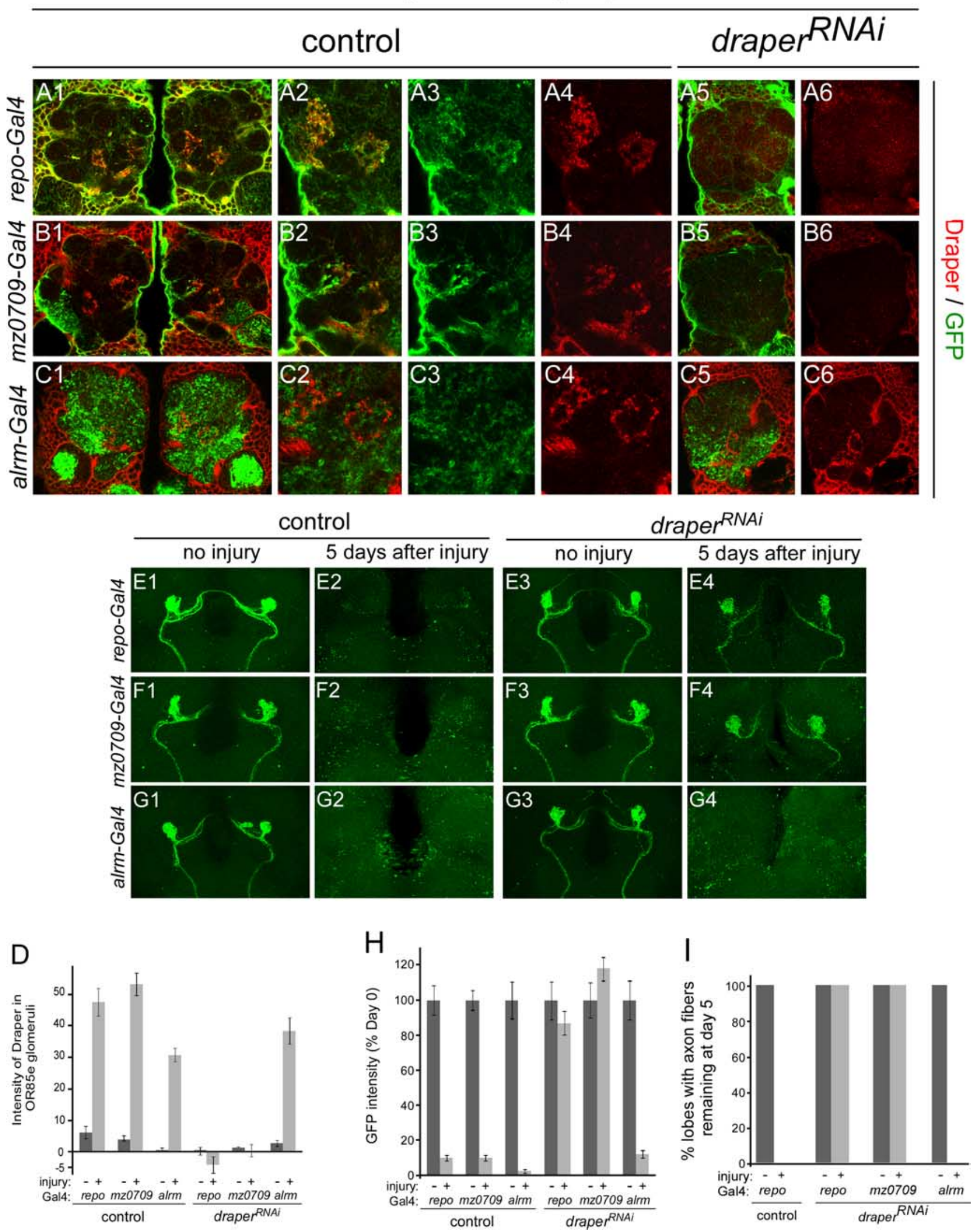
repo-Gal4 driver can be suppressed by coexpression of Gal80 (a Gal4 inhibitor) under control of the repo promoter (repo-Gal80) (Fig. 2A5), arguing that repo-Gal80 can efficiently block Gal4mediated activation of UAS-reporters in all adult brain glia.

Two drivers, mz0709-Gal4 and alrm-Gal4, appeared to show very specific expression in ensheathing glia and astrocytes, respectively (Fig. 2 B1,C1). Glial processes labeled by mz0709-Gal4 were found at the edge of the antennal lobe and extended deeply into the neuropil region (Fig. 2 B1,B2). These flattened glial processes surrounded, but did not invade, individual glomeruli (Fig. $2 B 3, B 4)$, and did not extend into the cortex region. With the exception of variable expression in a small number of neurons, all mz0709-Gal4-induced expression was suppressed by repo-Gal80, indicating that $m z 0709-G a l 4$ is largely specific to ensheathing glia. The generation of MARCM clones labeled with the $\mathrm{mz0709-}$ Gal4 driver resulted in the consistent labeling of ensheathing glia, but not astrocytes, within the antennal lobes. Reciprocally, alrmGal4 was found to be expressed exclusively in astrocytes (Fig. 2C). All cellular processes from cells labeled with alrm-Gal4 extended into the neuropil (Fig. 2C1,C2), showed a highly branched or tufted morphology, invaded individual glomeruli (Fig. 2C3,C4), and all alrm-Gal4-driven expression was suppressed by repoGal80 (Fig. 2C5). Additionally, we found that single cell MARCM clones labeled with the alrm-Gal4 driver resulted in the consistent labeling of astrocytes, but not ensheathing glia. Together, these drivers are excellent tools to manipulate and functionally distinguish different subtypes of glia in the adult Drosophila brain.

\footnotetext{
Figure 4. Ensheathing glia express Draper, are recruited to severed ORN axons, and phagocytose degenerating axonal debris. $\boldsymbol{A}-\boldsymbol{D}$, To assay recruitment of each glial subtype to severed axons, we ablated maxillary palps, allowed maxillary palp axons to degenerate for $1 \mathrm{~d}$, and then assayed glial membrane morphology with mCD8::GFP (green) and colocalization of glial membranes with Draper ( $\alpha$-Draper in red). Images are single confocal slices of the antennal lobe region. $\boldsymbol{A}$, In animals with Repo ${ }^{+}$glial membranes labeled with GFP we found that GFP was enriched in glomeruli housing degenerating maxillary palp ORN axons, that these same glomeruli were decorated with Draper, and that Repo ${ }^{+}$membranes colocalized perfectly with Draper (A1-A3). Expression of UAS-draper ${ }^{R N A i}$ in Repo ${ }^{+}$glia completely suppressed recruitment of glial membranes and Draper to severed axons $(\boldsymbol{A} 4, \boldsymbol{A} \boldsymbol{5}) . \boldsymbol{B}$, When glial membranes were labeled with $\mathrm{mCD}$-GFP using the $\mathrm{mz0709-Gal4}$ driver we found that $\mathrm{mz} 0709^{+}$GFP-labeled glial membranes also colocalized with Draper after maxillary palp ORNs were severed (B1-B3). Moreover, expression of UAS-draper ${ }^{R N A i}$ using the $m z 0709-G a l 4$ driver suppressed all recruitment of Draper and GFP-labeled membranes to severed axons $1 \mathrm{~d}$ after injury $(\boldsymbol{B} 4, \boldsymbol{B 5})$. $\boldsymbol{C}$, Labeling astrocyte membranes with mCD8::GFP using the alrm-Gal4 driver did not result in the colocalization of GFP-labeled glial membranes with severed axon-associated Draper staining (C1-C3), and expression of UAS-draper ${ }^{\text {RNAi }}$ using this driver failed to suppress Draper recruitment to severed axons $1 \mathrm{~d}$ after injury $(\boldsymbol{C} 4, \mathbf{C})$. D, Quantification of data from $\boldsymbol{A}$-C. Error bars represent SEM; $n \geq 10$ antennal lobes for each experiment. $\boldsymbol{E}-\boldsymbol{I}$, To explore the functional requirements for Draper in glial subtypes, we labeled a subset of ORN axons with OR85e-mCD8::GFP, drove UAS-draper ${ }^{\text {RNAi }}$ in glial subsets with the indicated drivers, severed axons by ablating maxillary palps, and assayed axon clearance $5 \mathrm{~d}$ after injury. All images are confocal Z-stack projections of GFP ${ }^{+}$OR85e axonal material. $\boldsymbol{E}-\mathbf{G}$, In control animals (no UASdraper $\left.^{R N A i}\right)$, axons developed normally $(\boldsymbol{E} \mathbf{1}, \boldsymbol{F} \mathbf{G}, \mathbf{G 1})$, degenerated, and were cleared from the CNS by $5 \mathrm{~d}$ after injury $(E 2, F 2, \mathbf{G 2})$. Driving UAS-draper ${ }^{R N A i}$ with repo-Gal4 $(E 3, E 4)$ or the ensheathing glial driver mz0709-Ga/4 $(\boldsymbol{F 3}, \boldsymbol{F 4})$ blocked glial clearance of severed axons. However, expression of UAS-draper ${ }^{R N A i}$ in astrocytes with alrm-Gal 4 had no effect on glial clearance of axons from the CNS 5 d after injury $(G 3, G 4)$. $\boldsymbol{H}$, Quantification of data from $\boldsymbol{E}-\boldsymbol{G}$. Error bars represent SEM; $n \geq 10$ antennal lobes for each experiment. $I$, The number of antennal lobes containing GFP-labeled axon debris $5 \mathrm{~d}$ after maxillary palp ablation was counted. In control animals (no UAS-DraperRNAi) no GFP-labeled axon debris remained $5 \mathrm{~d}$ after ablation. Knockdown of Draper with repo-Gal4 or mz0709-Gal4 resulted in GFP-labeled axon debris present in $100 \%$ of the antennal lobes counted $5 \mathrm{~d}$ after maxillary palp ablation. Consistent with control animals, driving UAS-draper ${ }^{R N A i}$ with alrm-Gal4 resulted in a complete absence of any GFPlabeled axons $5 \mathrm{~d}$ after injury. $n \geq 10$ antennal lobes for all.
}

\section{Ensheathing glia, but not astrocytes, express the engulfment receptor Draper}

What are the functional roles for each glial subtype in the adult brain? Is each subtype responsible for a unique collection of tasks, or are all glial subtypes functionally equivalent? As a first step to determining the in vivo functional differences between adult brain glial subtypes we explored the cell autonomy of glial phagocytic function. Severing olfactory receptor neuron (ORN) axons by surgical ablation of maxillary palps leads to axon degeneration (termed Wallerian degeneration), recruitment of glial membranes to fragmenting axons, and glial engulfment of axonal debris. These glial responses are mediated by Draper, the Drosophila ortholog of the C. elegans cell corpse engulfment receptor cell death defective-1 (CED-1). In draper null mutants, glia fail to extend membranes to degenerating ORN axons and axonal debris is not removed from the CNS (MacDonald et al., 2006). Thus, Draper function should be autonomously required in phagocytic glial subtypes and Draper expression is predicted to act as a molecular marker for glial cells capable of performing engulfment functions.

To define the precise cell types that express Draper, we first labeled all glial membranes with mCD8::GFP driven by repoGal4, stained with $\alpha$-Draper antibodies, and assayed for colocalization of Draper and GFP (Fig. 3). As previously reported, we found extensive overlap of Draper and GFP in this background (MacDonald et al., 2006). Draper and GFP signals overlapped at the edge of the neuropil, in membranes surrounding antennal lobe glomeruli, and in all cortex glia (Fig. 3A1-A3). This labeling was specific to Draper since expression of a UAS-draper ${ }^{R N A i}$ construct with repo-Gal4 led to the elimination of all Draper immunoreactivity in the adult brain (Fig. 3A4,A5). Thus, the entire population of cortex glia appear to express Draper and are likely to be phagocytic. However, cortex glia do not extend membranes into the antennal lobe neuropil, even after ORN axon injury (data not shown). Therefore, cortex glia are not likely responsible for clearing severed ORN axonal debris from the antennal lobe neuropil.

Interestingly, when mCD8::GFP was driven by mz0709-Gal4 we observed extensive overlap of Draper and GFP in neuropilassociated ensheathing glia (Fig. 3B1-B3). A high-magnification view of the antennal lobe revealed Draper and mz0709-Gal4 labeled membranes colocalizing and surrounding, but not innervating individual glomeruli (Fig. 3D1-D3). Moreover, expression of UAS-draper ${ }^{R N A i}$ in ensheathing glia with mz0709-Gal4 led to a dramatic reduction in Draper immunoreactivity in the neuropil, but the weaker Draper immunoreactivity in the cortex remained unchanged (Fig. 3B4,B5). Conversely, we observed no overlap between Draper and GFP when we labeled astrocytic membranes using the alrm-Gal4 driver (Fig. 3C1-C3,E1-E3). Furthermore, driving the expression of UAS-draper ${ }^{R N A i}$ in astrocytes had no obvious effect on Draper expression in the brain (Fig. 3C4,C5). These results indicate that Draper is expressed in cortex glia and ensheathing glia but not in astrocytes.

\section{Ensheathing glia use Draper to extend membranes to degenerating axons and engulf axonal debris}

The specific expression of Draper in antennal lobe ensheathing glia suggests that this glial subset is the phagocytic cell type responsible for engulfing degenerating axonal debris after ORN axotomy. To explore this possibility, we asked whether ensheathing glia or astrocytes extend membranes to severed axons after injury, and in which cell type Draper was required for clearance of axonal debris from the CNS. To assay extension of glial mem- 
branes to severed axons, we labeled glial membranes with mCD8::GFP, severed maxillary palp axons, and assayed for colocalization of Draper and GFP in glomeruli housing severed ORN axons. Within $1 \mathrm{~d}$ after injury, Repo ${ }^{+}$glial membranes were found to localize to glomeruli housing severed maxillary palp axons and these membranes were decorated with Draper immunoreactivity (Fig. 4A1-A4). Similarly, we found that $\mathrm{zz}_{0709^{+}}$ glial membranes also localized to severed axons and colocalized with intense Draper immunoreactivity $1 \mathrm{~d}$ after injury (Fig. 4 B1B4). Knockdown of Draper with UAS-draper ${ }^{R N A i}$ using repoGal4 or mz0709-Gal4 completely suppressed the recruitment of both Draper and glial membranes to severed axons (Fig. $4 A 5, A 6, B 5, B 6, D)$. In contrast, when astrocyte membranes were labeled with GFP we did not observe colocalization of GFP and Draper immunoreactivity $1 \mathrm{~d}$ after axotomy (Fig. 4C1-C4). In addition, knockdown of Draper in astrocytes with UASdraper $^{R N A i}$ did not suppress the recruitment of Draper to severed axons (Fig. $4 C 5, C 6, D)$. In an effort to identify any indirect role for astrocytes during the injury response, we examined the morphology of astrocytes both before and after injury to determine whether they exhibited any overt changes in morphology or retracted their membranes from the site of injury to accommodate the recruitment of ensheathing glial membranes. However, we did not detect any obvious changes in morphology or in the positions of the astrocyte glial cells in response to axon injury. Together, these data indicate that Draper is required in ensheathing glia for recruitment of glial membranes and accumulation of Draper on severed ORN axons, and suggest that Drosophila astrocytic glia do not undergo any dramatic changes in morphology in response to ORN axotomy.

From the above data we predicted that ensheathing glia would act as phagocytes to engulf degenerating ORN axonal debris from the CNS. To test this we labeled a subset of maxillary palp ORN axons with mCD8::GFP using the OR85e-mCD8::GFP transgene, knocked down Draper function in glial subsets using our subsetspecific driver lines, severed maxillary palp ORN axons, and assayed clearance of axons $5 \mathrm{~d}$ after injury. We first severed GFPlabeled axons in control animals with each driver and found that $\mathrm{GFP}^{+}$axonal debris was efficiently cleared from the CNS within $5 \mathrm{~d}$ after injury (Fig. 4E1,E2,F1,F2,G1,G2,H,I), confirming that glial phagocytic function is not affected in the driver lines. Strikingly, RNA interference (RNAi) knockdown of Draper using $U A S$-draper ${ }^{R N A i}$ in a background with repo-Gal4 or mz0709-Gal4 completely blocked clearance of GFP-labeled axonal debris from the CNS (Fig. 4E3,E4,F3,F4,H,I), while RNAi knockdown of Draper in astrocytes with alrm-Gal4 had no effect on axon clearance (Fig. 4G3,G4,H,I). Thus, Draper is required autonomously in ensheathing glia for the clearance of degenerating ORN axonal debris from the CNS. In addition, knockdown of Draper in ensheathing glia with $m z 0709-G a l 4$ had no measurable effect on Draper expression in cortex glia (see Fig. 3B4,B5), arguing that cortex glia are not capable of compensating for the loss of phagocytic activity in ensheathing glia during the clearance of axonal debris from the antennal lobe neuropil after axotomy. From these data on morphogenic responses to injury and phagocytic function, we conclude that astrocytic, cortex, and ensheathing glia represent functionally distinct subsets of glial cells in the adult Drosophila brain.

\footnotetext{
Shark, a Src-family kinase acting downstream of Draper, is required in ensheathing glia for clearance of degenerating axons We have recently shown that Shark, a non-receptor tyrosine kinase similar to mammalian Syk and Zap-70, is part of the Draper
}

signaling cascade and is essential to initiate phagocytic signaling events downstream of Draper during the engulfment of degenerating axons (Ziegenfuss et al., 2008). Our model that Draper functions exclusively in ensheathing glia for clearance of degenerating ORN axons predicts that Shark and other components of the Draper signaling cascade would also function in ensheathing glia. To determine whether Shark function is required in ensheathing glia, we knocked down Shark in glial subsets and assayed the recruitment of Draper to severed ORN axons $1 \mathrm{~d}$ after injury, and the clearance of degenerating axonal debris from the CNS $5 \mathrm{~d}$ after axotomy. Consistent with a role for Shark in ensheathing glia, we found that knockdown of Shark with UAS$S_{\text {Shark }}^{R N A i}$ (Ziegenfuss et al., 2008) driven by repo-Gal4 or mz0709-Gal4 strongly suppressed both the recruitment of mCD8::GFP-labeled glial membranes and Draper to severed axons (Fig. $5 A 1, A 2, B 1, B 2, D$ ), as well as the clearance of degenerating axonal debris from the CNS (Fig. 5E1,E2,F1,F2, H,I). However, knockdown of Shark with alrm-Gal4 had no effect on the recruitment of Draper or GFP-labeled glial membranes to degenerating axons (Fig. 5C1,C2,D), nor did it inhibit the clearance of axonal debris from the CNS (Fig. 5G1,G2,H,I). Thus, Shark, like Draper, is required in ensheathing glia for efficient extension of glial membranes to degenerating axons and clearance of degenerating axonal debris from the CNS.

\section{dCed-6 is expressed in ensheathing and cortex glia and is required for glial clearance of degenerating axons}

In C. elegans the PTB domain adaptor protein CED-6 acts genetically downstream of CED-1 during engulfment of apoptotic cells (Liu and Hengartner, 1998) and, during Drosophila metamorphosis, RNAi knockdown of dCed-6 has been shown to partially suppress glial engulfment of pruned axon arbors during remodeling of larval mushroom body $\gamma$ neurons (Awasaki et al., 2006). We asked whether dCed- 6 was involved in glial responses to axon injury in the adult brain. First, to determine where dCed-6 is expressed in the adult CNS we stained control animals with Draper and dCed-6 antibodies and found that Draper and dCed-6 immunoreactivity perfectly overlapped throughout the adult brain (Fig. $6 A 1, B 1, C 1, D 1-D 4$ ). Since we have shown that Draper is expressed in cortex and ensheathing glia, we conclude that dCed-6 is also expressed in these glial subtypes.

Next we asked whether dCed-6 was recruited to degenerating ORN axons. We performed maxillary palp or antennal ablations and compared dCed- 6 and Draper recruitment $1 \mathrm{~d}$ after injury. We consistently found that dCed-6 colocalized with Draper at sites of severed axons after maxillary palp (Fig. 6A2,B2,C2) and antennal injury (Fig. 6A3,B3,C3), which is consistent with dCed- 6 being expressed in ensheathing glia and possibly functioning downstream of Draper.

To obtain genetic evidence that dCed- 6 was essential for glial clearance of degenerating axonal debris, we used the $D f(2 R) w 73-1$ deletion chromosome, which harbors a small deletion that removes the dCed- 6 gene, and assayed for genetic interactions between this dCed- 6 deletion chromosome and draper mutants. In control animals, GFP-labeled ORN axons are largely cleared $3 \mathrm{~d}$ after injury (Fig. 6E1,E2,I). Interestingly, we found that while the majority of axons were cleared at this time point in draper $^{\Delta 5} /+$ or $D f(2 R) w 73-1 /+$ animals (Fig. $6 F 1, F 2, G 1, G 2, I$ ), a significant number of axons remained in $D f(2 R) w 73-1 /+$; drap$e r^{\Delta 5} /+$ transheterozygous animals (Fig. $6 \mathrm{H1}, \mathrm{H} 2, \mathrm{I}$ ). These data are consistent with draper and ced-6 exhibiting strong genetic interactions during glial clearance of degenerating axons.

The expression pattern of dCed- 6 in the adult brain suggests 

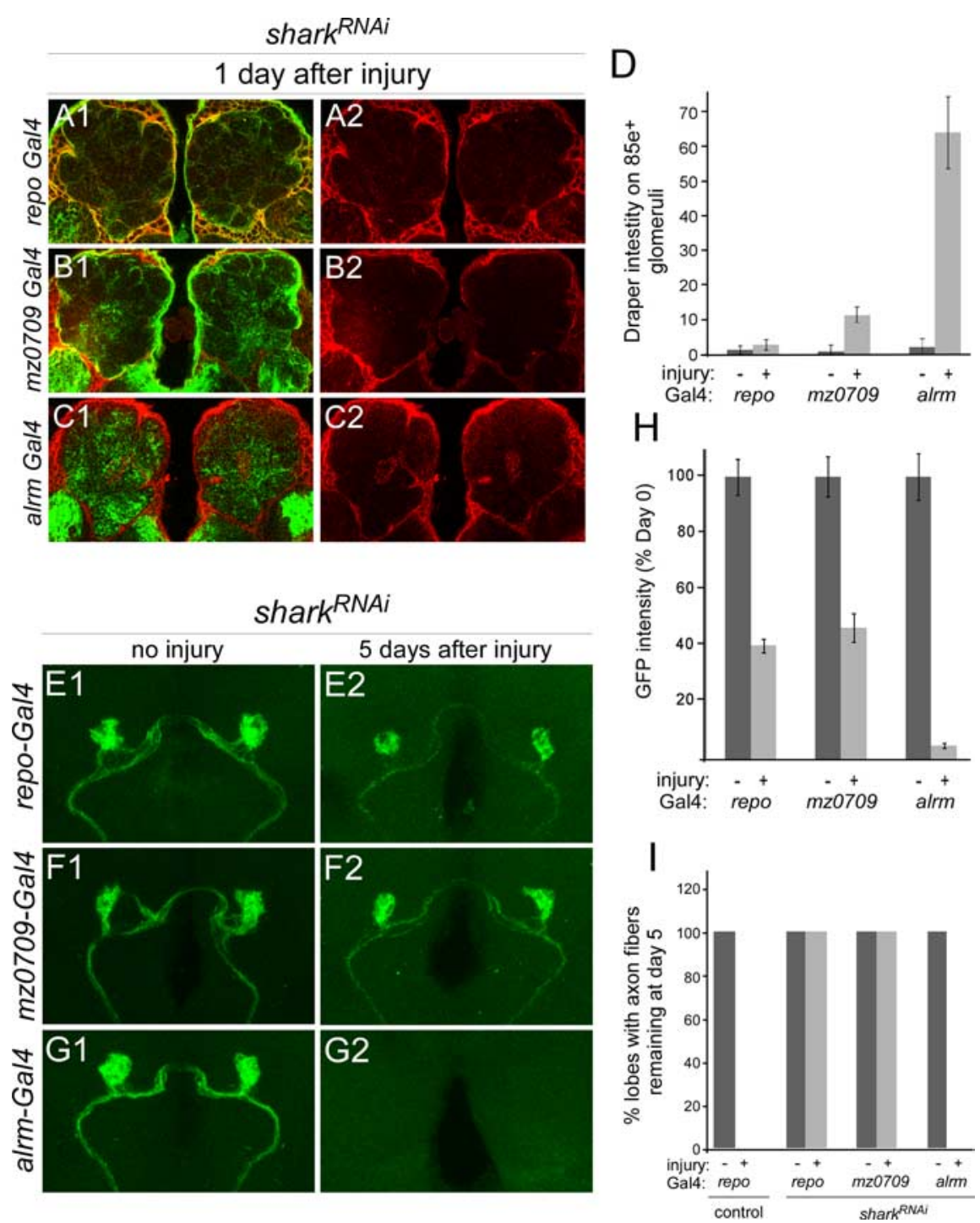

Figure 5. The non-receptor tyrosine kinase Shark functions in ensheathing glia to drive engulfment of ORN axonal debris. We knocked down Shark function in subsets of glia with UAS-Shark ${ }^{R A i}$ and assayed recruitment of Draper to severed maxillary palp axons $1 \mathrm{~d}$ after injury $(\boldsymbol{A}-\boldsymbol{D})$, and glial engulfment of degenerating axonal debris $5 \mathrm{~d}$ after injury $(\boldsymbol{E}-\boldsymbol{I}) . \boldsymbol{A} \mathbf{1}, \boldsymbol{B} \mathbf{1}$, and $\mathbf{C} \mathbf{1}$ show $\alpha$-Draper (red) and Gal4-driven mCD8::GFP (green); $\boldsymbol{A 2}, \mathbf{B 2}, \mathbf{C}$ show Draper alone. Representative single confocal slices ( $\boldsymbol{A}-\boldsymbol{C})$ and confocal Z-stacks $(\boldsymbol{E}-\boldsymbol{G})$ are shown. $\boldsymbol{A}$, Knockdown of Shark in Repo ${ }^{+}$glia suppressed recruitment of Draper to severed maxillary palp ORN axons $1 \mathrm{~d}$ after injury. $\boldsymbol{B}$, Knockdown of Shark in ensheathing glia with mz0709-Gal4 also suppressed recruitment of Draper to severed axons. $C$, Draper was strongly recruited to severed axons when UAS-Shark ${ }^{R N A i}$ was driven in astrocytes with alrm-Gal4. D, Quantification of data from $\boldsymbol{A}-\boldsymbol{C}$. Error bars represent SEM; $n \geq 10$ antennal lobes for each experiment. $\boldsymbol{E}$, Shark ${ }^{\mathrm{RNAi}}$ in Repo ${ }^{+}$glia suppressed glial clearance of degenerating axonal debris. $\boldsymbol{F}$, Knockdown of Shark in ensheathing glia also suppressed glial clearance of degenerating axonal debris. G, Shark ${ }^{\text {RNAi }}$ treatment of astrocytes failed to suppress glial clearance of degenerating axonal debris. $\boldsymbol{H}$, Quantification of data from $\boldsymbol{E}-\boldsymbol{G}$. Error bars represent SEM; $n \geq 10$ antennal lobes for each experiment. $\boldsymbol{I}$, The percentage of antennal lobes containing GFP ${ }^{+}$axonal membranes $5 \mathrm{~d}$ after maxillary palp ablation. In contro animals (no UAS-shark ${ }^{R N A i}$ ), no GFP + axonal material was present in any samples. Shark ${ }^{\text {RNAi }}$ treatment of all glial cells, using repo-Gal4, or ensheathing glial cells, using mz0709-Gal4, resulted in perdurance of GFP ${ }^{+}$axonal debris $5 \mathrm{~d}$ after maxillary palp ablation in $100 \%$ of the samples. Similar to control animals, driving UAS-shark ${ }^{R N A i}$ with alrm-Gal4 led to a complete loss of GFP ${ }^{+}$ axonal material $5 \mathrm{~d}$ after injury in all antennal lobes. $n \geq 10$ antennal lobes for all.

that it is acting in ensheathing and/or cortex glia to mediate Draper-dependent glial engulfment functions. To determine the autonomy of dCed- 6 function in the adult brain, we knocked down dCed-6 in different glial subsets using a UAS-dced- $6^{R N A i}$ construct and assayed Draper recruitment to severed axons and clearance of degenerating axonal debris (Fig. 7). Knockdown of dCed-6 in all glial cells using repo-Gal4 suppressed both the recruitment of Draper to the site of injury (Fig. $7 A 1, A 2, B 1, B 2, E$ ) as well as the clearance of degenerating $\mathrm{GFP}^{+}$axon material $5 \mathrm{~d}$ after injury compared with control animals (Fig. 7F1,F2,G1,G2,J). Based on our findings using Draper ${ }^{\text {RNAi }}$ and Shark ${ }^{\text {RNAi }}$, we expected Ced- $6^{\text {RNAi }}$ treatment using the mz0709-Gal4 driver to also suppress glial responses to axon injury. However, we found normal levels of Draper recruited to severed axons $1 \mathrm{~d}$ after injury (Fig. 7C1,C2,E) and efficient clearance of axon material $5 \mathrm{~d}$ after injury (Fig. $7 \mathrm{H} 1, \mathrm{H} 2, \mathrm{~J})$. To assay the level of dCed-6 knockdown by RNAi, we stained brains with $\alpha$-dCed- 6 antibodies $5 \mathrm{~d}$ after maxillary palp ablation and found moderate levels of dCed-6 staining when UAS-dced$6^{R N A i}$ was driven by mz0709-Gal4 or alrmGal4, but not when driven by repo-Gal4 (Fig. 7F3,G3,H3,I3). Thus, mz0709-Gal4 does not appear to provide a complete knockdown of dCed-6 in ensheathing glia. Nevertheless, based on the colocalization of Draper, and dCed- 6 in ensheathing glial subtypes in the adult brain, we propose that dCed-6 acts in ensheathing glia to promote Draper-dependent recruitment of glial membranes to severed axons and clearance of degenerating axonal debris.

\section{Blocking endocytosis in ensheathing glia suppresses glial clearance of severed axons}

Our analyses of Draper, Shark, and dCed-6 indicate that ensheathing glia express all components of the engulfment machinery and that the Draper signaling pathway is essential in ensheathing glia for efficient clearance of degenerating axons in the adult brain. These findings argue that ensheathing glia are the primary phagocytic cell type in the adult brain neuropil. Nevertheless, to further exclude any possible role for astrocytic glia in engulfing degenerating axons, we used the dominant temperature-sensitive shibire ${ }^{t s}$ molecule $\left(U A S-\right.$ shibire $\left.^{\text {ts }}\right)$ to conditionally block endocytosis in either astrocytes or ensheathing glia, and subsequently assayed glial responses to axon injury.

Interestingly, when we raised animals expressing shibire ${ }^{t s}$ under the control of repo-Gal4 at $18^{\circ} \mathrm{C}$ and then subsequently shifted the adult animals to the restrictive temperature of $30^{\circ} \mathrm{C}$, we observed $100 \%$ lethality within $3 \mathrm{~d}$ after temperature shift. Thus, suppressing glial endocytic function in all glia results in rapid adult lethality, indicating that in the healthy adult Drosophila brain glial cells likely perform a high level of endocytic events that are essential for viability.

We next drove shibire ${ }^{t s}$ in ensheathing or astrocytic glia and assayed recruitment of Draper to severed axons $1 \mathrm{~d}$ after injury. At the permissive temperature of $18^{\circ} \mathrm{C}$, expression of Shibire ${ }^{\text {ts }}$ $\left(\mathrm{Shi}^{\mathrm{ts}}\right)$ in ensheathing glia had no effect on Draper recruitment to severed axons. However, shifting animals to the restrictive tem- 


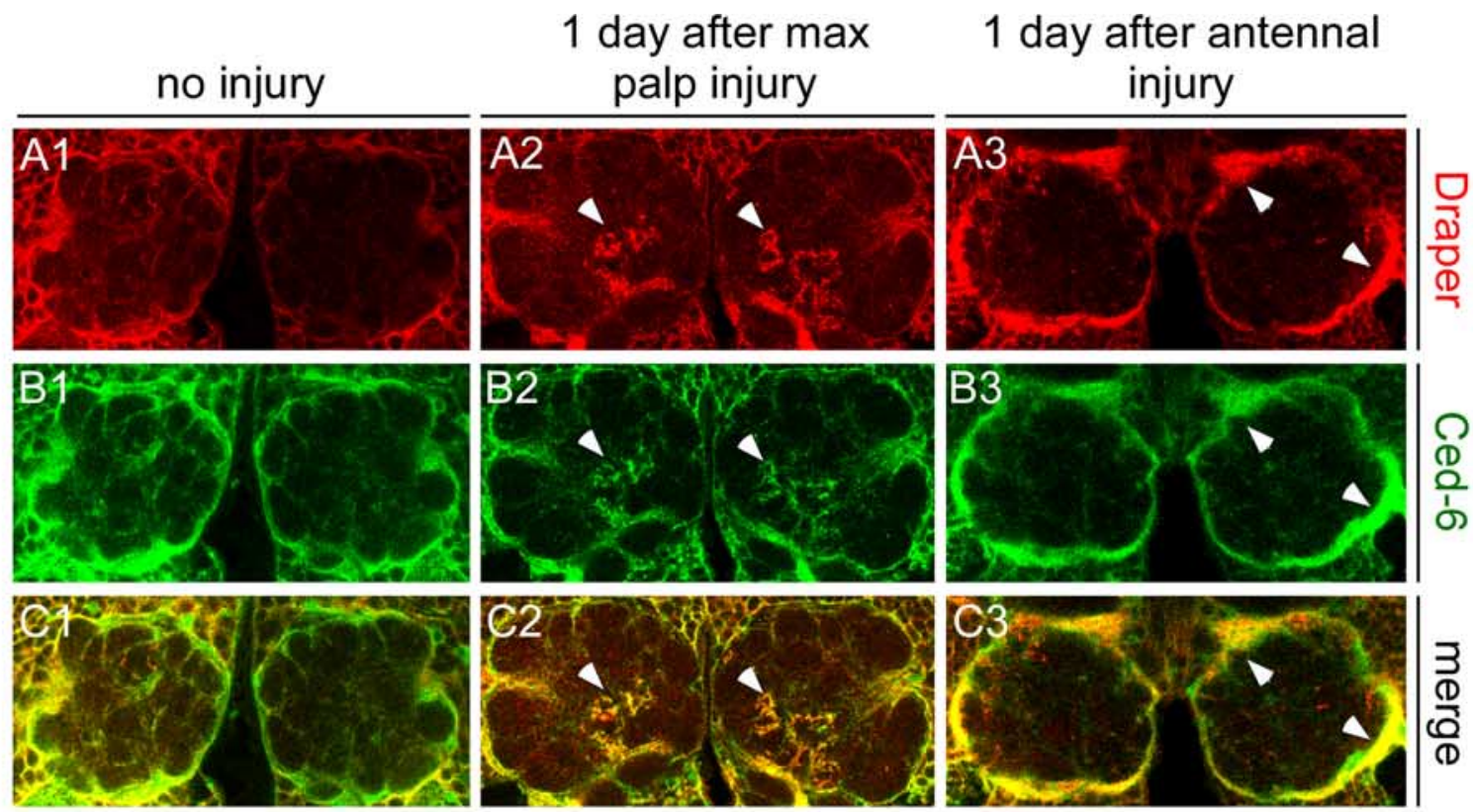

High magnification of single antennal lobe

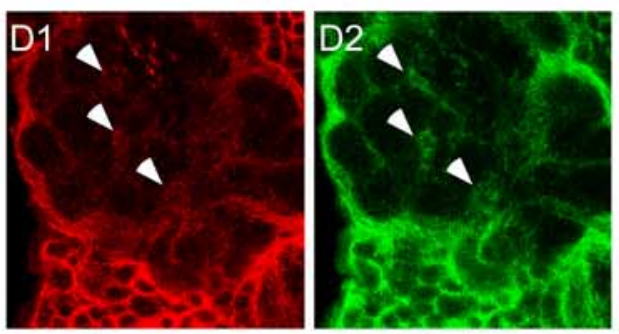

Draper
Ced-6

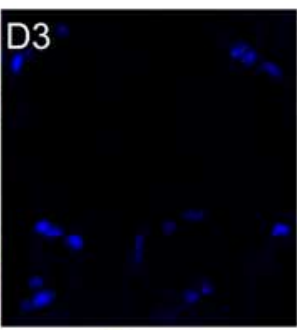

Repo

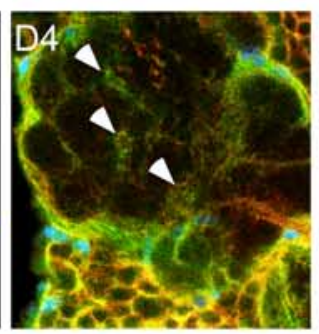

merge
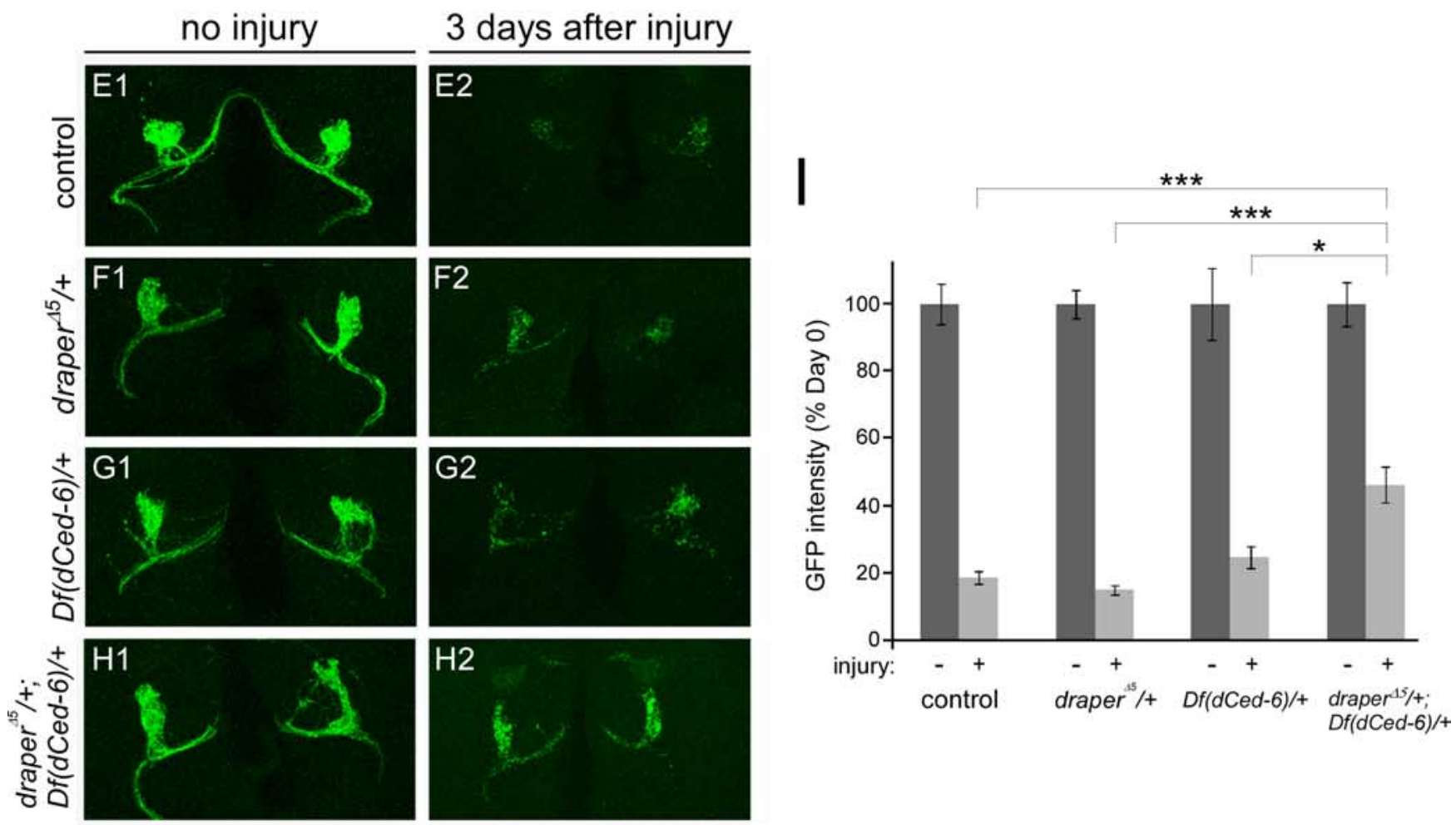
perature strongly suppressed this response (Fig. $8 A 1-A 4, C)$. In contrast, expression of $\mathrm{Shi}^{\mathrm{t}}$ in astrocytes had no effect on Draper recruitment to severed axons at either permissive or restrictive temperatures (Fig. 8B1-B4,C). Moreover, we found that blocking endocytic function in ensheathing glia with $\mathrm{Shi}^{\text {ts }}$ (at restrictive temperature) strongly suppressed glial clearance of degenerating axons from the brain, while the same treatment of astrocytes had no effect on axon clearance (Fig. 8D1$D 4, E 1, E 2, F, G)$. These data provide additional compelling evidence that ensheathing glia are the primary phagocytic cell type responsible for engulfing degenerating ORN axons. We also note, since blocking endocytic function in astrocytes did not affect glial clearance of severed axons (Fig. $8 E 3, E 4, F, G$ ), these data further argue that neither phagocytic activity nor signaling events involving endocytosis in astrocytes are essential for efficient clearance of degenerating axonal debris from the CNS.

\section{Discussion}

\section{Molecular and morphological subtypes of glia in the Drosophila adult brain}

We have identified three molecularly and morphologically distinct subtypes of glia in the adult Drosophila brain: ensheathing glia, astrocytes, and cortex glia. We note again that surface glia, which form the blood brain barrier, are another major glial subtype in the adult brain (Bainton et al., 2005; Schwabe et al., 2005), but surface glia do not make extensive contact with neurons and we have not addressed this subtype in this study. Rather we have focused only on those glial subtypes that are in direct association with CNS neurons.

The morphology of ensheathing glia, astrocytes, and cortex glia appears to be hardwired. For example, 100\% of astrocytic membrane processes invade the neuropil, and we never observed astrocyte processes straying into the cell cortex. Likewise, ensheathing glia and cortex glia appear to respect discrete spatial boundaries within the brain. Ensheathing glia extend the membranes into the neuropil, but not into the cell cortex, and within the neuropil they appear to primarily form boundaries between neuropilar structures with brain lobes such as antennal lobe glomeruli or different regions of the mushroom body (i.e., $\alpha / \beta$, $\alpha^{\prime} \beta^{\prime}$, and $\gamma$ lobes), or between discrete brain centers (e.g., antennal lobe and subesophageal ganglion). Cortex glia extend profuse

Figure 6. dCed-6 6 is recruited to severed $0 \mathrm{RN}$ axons after injury and genetically interacts with Draper. A-C, We compared $\alpha$-dCed-6 (green) and $\alpha$-Draper (red) localization in three sets of animals: control (no injury), $1 \mathrm{~d}$ postmaxillary palp ablation, and $1 \mathrm{~d}$ postantennal ablation. Representative images of single confocal slices through the antennal lobe regions are shown. Draper (A1) and dCed-6 (B1) had overlapping patterns of expression (C1) in control animals, including cortex and neuropil regions of the brain. One day after maxillary palp ablation, high levels of Draper (A2) and dCed-6 (B2) immunoreactivity were colocalized (C2) on severed axons (arrowheads). Ablation of antennae resulted in a characteristic dramatic increase in Draper immunoreactivity in glia surrounding the antennal lobes $1 \mathrm{~d}$ later $(\boldsymbol{A} \mathbf{3})$. We found that $\mathrm{dCed}-6$ also showed a dramatic increase in immunoreactivity in glia outlining the antennal lobes (white arrowheads) at this time point (B3, C3). D, Single confocal slice of antennal lobe at high magnification stained with $\operatorname{Draper}(\boldsymbol{D} 1)$, dCed-6 (D2), and Repo (D3), and merged image (D4). Note colocalization of Draper and dCed- 6 (arrowheads). $\boldsymbol{E}-\boldsymbol{I}$, To assay axon clearance of $85 \mathrm{e}^{+}$maxillary palp ORN axons were labeled with mCD8::GFP, maxillary palps were ablated, and the amount of GFP ${ }^{+}$axonal debris was quantified in control, draper ${ }^{\Delta 5} /+, D f(d(e d-6) /+$, and Df(dCed-6) $/+;$ draper $^{\Delta 5} /+$ animals 3 d after axotomy. Representative confocal Z-stackimages are shown. Severed axons were largely cleared $3 \mathrm{~d}$ after injury in control $(\boldsymbol{E})$, draper $^{\Delta 5} /+(\boldsymbol{F})$, and $D f(d(e d-6) /+(G)$ animals. Less axonal debris was cleared from the CNS $3 \mathrm{~d}$ after injury in $D f\left(d(e d-6) /+; d_{\text {raper }} \Delta 5 /+(\boldsymbol{H})\right.$ animals compared with all other genotypes. I, Quantification of data from $\boldsymbol{E}-\boldsymbol{H}$. Error bars represent SEM; $n \geq 10$ antennal lobes for each experiment; ${ }^{*} p<$ $0.05 ; * * * 00.0001$. projections throughout the region of the brain housing neuronal cell bodies, and, impressively, appear to individually enwrap each neuronal cell body, but we never observed cortex glia extending membranes out of their unique spatial domain (the cortex) and into the neuropil. We have never found an adult brain glial cell that has the characteristics of more than one glial subtype (e.g., a cell with membranes in the cortex surrounding cell bodies that also ensheaths an antennal lobe glomerulus). Thus the morphology of ensheathing, astrocytic, and cortex glial membranes is precisely controlled, presumably by underlying molecular programs that are specific to each subtype. In the future it will be exciting to determine how these unique glial subtypes, spatial domains, and morphologies are established, and to identify the underlying molecular pathways that govern glial subtype identity and morphogenesis. In addition, we may find that some or all of these glial subtypes can be further refined into smaller populations, just as it has long been thought that astrocytes comprise heterogeneous populations of glia (Matthias et al., 2003; Barres, 2008).

Importantly, while the gross morphology and spatial domain of any particular glial subtype is highly stereotyped, the precise morphology of each glial cell within any given class may not be preprogrammed or stereotyped. For example, in our MARCM analysis of ensheathing glia or astrocytes, each single cell clone had a unique morphology (i.e., we did not observe specific ensheathing glia or astrocytes based on position or morphology that were uniquely identifiable from animal to animal), and the position of glial nuclei and glial cell bodies at the edge of the neuropil appeared variable from one animal to the next. We suspect the precise morphology of glia within these classes is determined by cell-cell trophic interactions with neighboring glia of the same class. Our observations that each glial subtype (cortex, ensheathing, or astrocytic) distributes membranes throughout its entire spatial domain argues that regulatory pathways are in place to ensure full coverage of each spatial domain with the appropriate glial subtype. Finally, in support of our conclusion on the stereotyped morphologies of these glial populations we note that Awasaki et al. (2008) have recently described a very similar complement of glial subtypes using MARCM clonal analysis in the Drosophila adult brain.

\section{Drosophila adult brain glial subtypes have unique functions in the brain}

Mammalian glial subtypes are thought to play specific roles in CNS development, function, and health. Astrocytes are general regulators of synapse formation and physiology. They secrete synaptogenic molecules, associate closely with mature synapses, buffer ions, $\mathrm{pH}$, and neurotransmitters at the synaptic cleft, and likely modulate synaptic efficacy and signaling. Oligodendrocytes physically separate axons through ensheathment, and myelinate them to allow for saltatory conduction. Microglia are the resident immune cells of the brain, rapidly responding to injury, phagocytosing dead cells and cellular debris and modulating brain inflammatory responses (Barres, 2008). Our work demonstrates that a diversity of glial subtypes also exist in the adult Drosophila brain, each with unique morphological, functional, and molecular attributes.

As a first step in defining the functional differences between glial subtypes in the adult Drosophila brain we focused primarily on the role of ensheathing glia and astrocytes in engulfing degenerating axons after brain injury. We have shown that ensheathing glia express all key components of the Draper signaling pathway (see below), respond morphologically to axon injury by extend- 
ing membranes to severed axons, and clear degenerating ORN axons from the brain. Thus ensheathing glia appear to be the primary cell type that clears degenerating axonal debris from the neuropil and plays the role of resident phagocyte. Moreover, since ensheathing glia invade new spatial regions of the brain after injury (e.g., antennal lobe glomeruli) we cannot rule out the possibility that ensheathing glia extend membranes into the cell cortex and engulf neuronal debris after cell cortex-specific axotomy or neuronal death. However, cortex glia may also perform phagocytic functions in the cell cortex region of the brain since they express engulfment genes (Draper and dCed-6). Unfortunately, we are unable to test these ideas directly since we are currently unable to generate reproducible brain lesions in the cell cortex.

How do ensheathing glia survey the brain for axonal injury? With respect to the olfactory system, ensheathing glia envelop both the antennal and maxillary nerves as they enter the brain and their membranes are in close proximity to axons up to the point where axons enter to terminate within individual antennal lobe glomeruli. Upon axotomy, ensheathing glia are thus uniquely positioned to sense "eat me" cues presented by degenerating ORN axons. Since ensheathing glial membranes also invade antennal lobe glomeruli, a region of the brain where they normally are absent, these axon-derived cues are clearly sufficient to drive dynamic extension of ensheathing glial membranes into new regions of the CNS.

Of the three subtypes of glia we describe the Drosophila astrocyte is most striking in its resemblance to its mammalian counterpart. Similar to mammalian protoplasmic astrocytes, Drosophila astrocytes show a highly branched and tufted morphology, extending profuse membrane specializations into regions of the fly brain that are rich in synapses. At the molecular level, these cells also appear similar to mammalian astrocytes: fly astrocytes express the high-affinity excitatory amino acid transporter, EAAT1, homologous to mammalian GLAST and GLT-1, which

transport the glutamate into glial cells where it is then converted into glutamine by glutamine synthetase (supplemental Fig. 1, available at www.jneurosci.org as supplemental material). Our identification and characterization of the astrocyte-specific alrmGal4 driver now opens the door to exciting genetic, cellular, and molecular analyses of astrocyte development and function in Drosophila.

We found no evidence that fly astrocytes in the adult brain respond to axon injury, or are required for clearance of degenerating axons from the CNS. Drosophila astrocytes exhibit no detectable changes in morphology after ORN axon injury, lack en- dced-6 $6^{R N A i}$

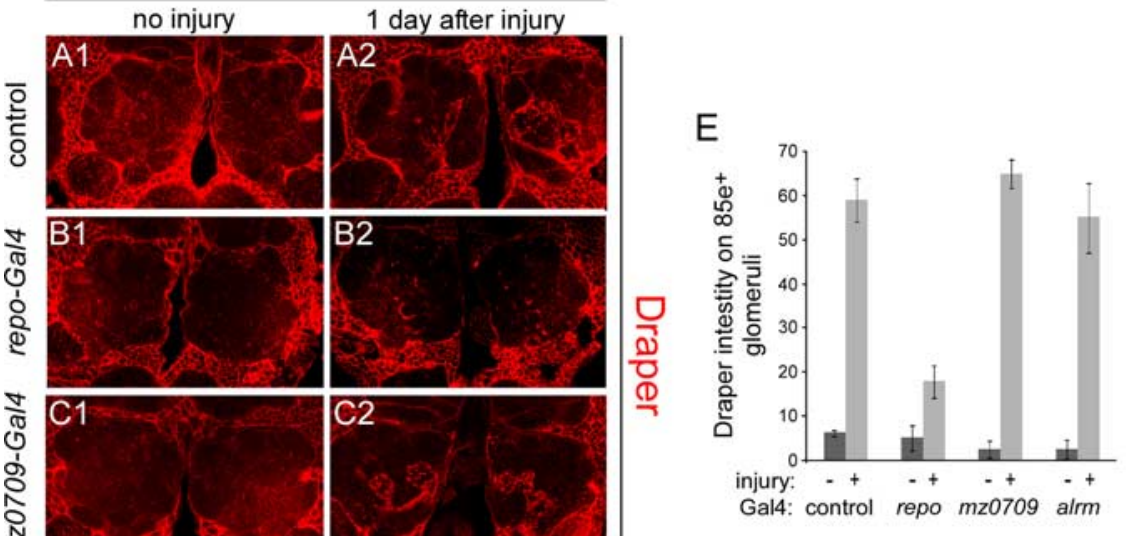

$\mathrm{J}$

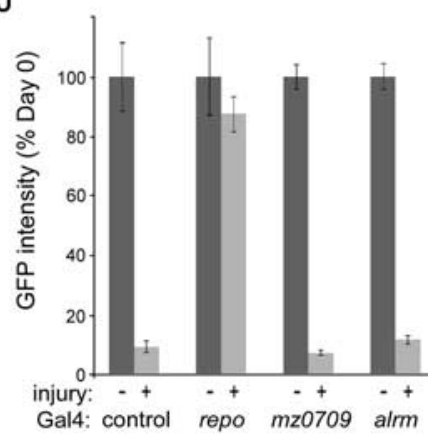

Figure 7. Glial-specific knockdown of dCed-6 suppresses clearance of degenerating ORN axonal debris. dCed-6 was knocked down in glia using a UAS-dced- $6^{\text {RNAi }}$ construct and recruitment of Draper to severed axons $(\boldsymbol{A}-\boldsymbol{E})$ and clearance of degenerating $\mathrm{GFP}^{+}$axonal debris $(\boldsymbol{F}-\boldsymbol{J})$ were assayed. Representative single confocal images $(\boldsymbol{A}-\boldsymbol{D})$ and $Z$-stack projections $(\boldsymbol{F}-\boldsymbol{I})$ are shown. $\boldsymbol{A}$, In control animals (repo-Gal4 driver alone), Draper was recruited at high levels to severed axons $1 \mathrm{~d}$ after injury. $\boldsymbol{B}$, Pan-glial knockdown of dCed-6 using repo-Gal 4 suppressed Draper recruitment to severed axons. C, Knockdown of dCed-6 in ensheathing using alrm-Gal 4 did not affect recruitment of Draper to severed axons after axotomy. E, Quantification of data from $\boldsymbol{A}-\boldsymbol{D}$. Error bars represent SEM; $n \geq 10$ antennal lobes for each experiment. $\boldsymbol{F}$, In control animals (repo-Gal4 driver alone), severed axons were cleared from the CNS 5 d after injury $(\boldsymbol{F 1}, \boldsymbol{F 2})$, and dCed-6 was expressed strongly throughout the adult brain $(\boldsymbol{F} \mathbf{3}) . \mathbf{G}$, Knockdown of dCed-6 in all glia with repo-Gal4 completely suppressed clearance of degenerating axons $5 \mathrm{~d}$ after injury $(\mathbf{G 1}, \mathbf{G 2})$, and dCed-6 immunoreactivity was no longer detectable in the adult brain (G3). $\boldsymbol{H}$, Knockdown of dCed-6 in ensheathing glia with $\mathrm{mz} 0709$ Gal4 did not suppress glial clearance of axonal debris $(\boldsymbol{H 1}, \boldsymbol{H 2})$, and, notably, dCed-6 staining in the adult brain was only partially reduced in dCed-6 ${ }^{\text {RNAi }}$ animals (white arrowheads in $\boldsymbol{H 3}$ ). $\boldsymbol{I}, \mathrm{dCed}-6$ knockdown in astrocytes with alrm-Gall had no effect on clearance of axonal debris $(\boldsymbol{I 1}, \mathbf{I})$ or dCed-6 staining in the adult brain $(\boldsymbol{I 3})$. J, Quantification of axon clearance data from $(\boldsymbol{F}-\boldsymbol{I})$. $n \geq 10$ antennal lobes for each experiment, error bars are SEM.

dogenous expression of components of the Draper signaling pathway, and they do not appear to activate the expression of these genes after axon injury. Moreover, we found that knocking down Draper signaling or blocking the majority of endocytic function in astrocytes with shibire ${ }^{\text {ts }}$ had no effect on the clearance of degenerating axons by ensheathing glia. A number of transmembrane receptors mediating cell-cell signaling require endocytosis for signaling events. The latter result therefore argues that such pathways in astrocytes must be dispensable for ensheathing glial clearance of degenerating axons from the CNS. These observations were somewhat surprising since mammalian astrocytes 

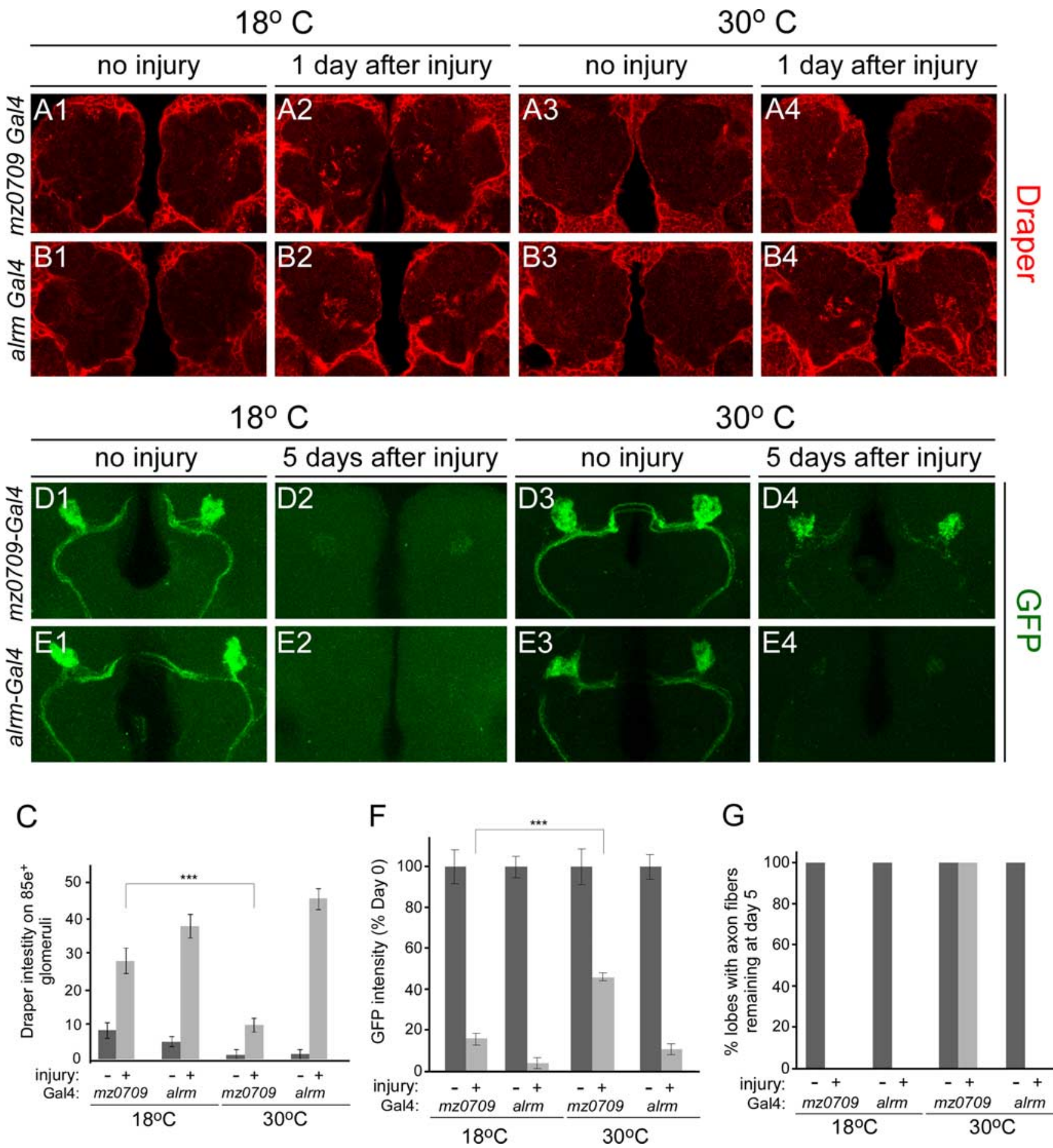

Figure 8. Endocytic function is required in ensheathing glia, but not astrocytes, for glial clearance of degenerating ORN axons. The requirements for endocytic activity during phagocytosis of axons in ensheathing glia and astrocytes were determined by expressing UAS-shibire ${ }^{\text {ts }}$ with mz0709-Gal4 and alrm-Gal4, respectively, and assaying recruitment of Draper to severed axons and clearance of degenerating axonal debris from the brain. $A$, Expression of Shibire ${ }^{t s}$ in ensheathing glia did not block recruitment of Draper to severed maxillary palp $0 \mathrm{RN}$ axons $1 \mathrm{~d}$ after injury when animals were maintained at the permissive temperature of $18^{\circ} \mathrm{C}(\boldsymbol{A} \mathbf{1}, \boldsymbol{A} \mathbf{2})$, but Draper recruitment to severed axons was strongly suppressed when these animals were maintained at the restrictive temperature of $30^{\circ} \mathrm{C}(\boldsymbol{A} \mathbf{B}, \boldsymbol{A 4}) \cdot \boldsymbol{B}$, Shibire ${ }^{\text {ts }}$ expression in astrocytes had no effect on Draper recruitment to severed axons at either $18^{\circ} \mathrm{C}$ or $30^{\circ} \mathrm{C}(\boldsymbol{B} 1-\boldsymbol{B} 4)$. C, Quantification of data from $\boldsymbol{A}$ and $\boldsymbol{B}$. Error bars represent SEM; $n \geq 10$ antennal lobes for each experiment. ${ }^{* * *} p<0.0001$. D. Expression of Shibire ${ }^{\text {ts }}$ in ensheathing glia had no effect on glial clearance of severed axons at $18^{\circ} \mathrm{C}(\boldsymbol{D 1}$, D2). Shifting to the restrictive temperature of $30^{\circ} \mathrm{C}$ strongly suppressed clearance of degenerating axons $5 \mathrm{~d}$ after axotomy $(\mathbf{D}, \mathbf{D} 4)$. $\boldsymbol{E}$, Expression of Shibire ${ }^{\text {ts }}$ in astrocytes with alrm-Gal4 had no effect on glial clearance of degenerating axons $18^{\circ} \mathrm{C}$ or $30^{\circ} \mathrm{C}(\boldsymbol{E} 1-\boldsymbol{E} 4) . \boldsymbol{F}$, Quantification of data from $\boldsymbol{D}$ and $\boldsymbol{E}$. Error bars represent SEM; $n \geq 10$ antennal lobes for each experiment; ${ }^{* * *} p<0.0001$. G, The number of antennal lobes containing GFP-labeled axon debris $5 \mathrm{~d}$ after maxillary palp ablation were counted and expressed as a percentage of the total number. In control animals (entire duration of the experiment performed at the permissive temperature of $18^{\circ} \mathrm{C}$ ) no GFP-labeled axons were present in either the $\mathrm{mz} 0709-\mathrm{Gal} 4 \mathrm{or}$ the alrm-Gal 4 flies. When the flies are shifted to the restrictive temperature of $30^{\circ} \mathrm{C}, 100 \%$ of the axons in the $\mathrm{mz0709-Gal4}$ flies are still GFP ${ }^{+}$, whereas none of the axons in the alrm-Gal4 flies contain any GFP-labeled axons. 
respond to a variety of brain injury by increasing expression of glial fibrillary acid protein (GFAP) and undergoing hypertrophy (Murray et al., 1990; Liu et al., 1998) and intercellular ATP signaling occurs between astrocytes and microglia in the mouse cortex after acute injury (Davalos et al., 2005). Since a GFAP gene is not present in the Drosophila genome we were unable to assay its expression, but fly astrocytes showed no obvious morphological changes after injury, suggesting they do not undergo axon injury-induced hypertrophy. A major proposed role for reactive astrocytes in mammals is the modulation of neuroinflammation (Wyss-Coray and Mucke, 2002), rather than phagocytic activity. Since classical tissue inflammatory responses have not been described in Drosophila, modulation of neuroinflammation may be an astrocytic function specific to more complex nervous systems than those found in Diptera.

\section{Ensheathing glia express components of the Draper engulfment signaling pathway and act as phagocytes to clear degenerating ORN axons}

The Draper signaling pathway is a central mediator driving glial engulfment of neuronal cell corpses (Freeman et al., 2003), pruned axons and dendrites (Awasaki et al., 2006; Hoopfer et al., 2006; Williams et al., 2006), and axons undergoing Wallerian degeneration (MacDonald et al., 2006) in Drosophila. Our studies show that within the adult brain neuropil, ensheathing glial cells are the only cell type that expresses Draper. Draper expression overlaps precisely with ensheathing glial membranes (when labeled with GFP driven by the ensheathing glia-specific driver mz0709-Gal4), and RNAi for draper in ensheathing glia leads to the elimination of Draper immunoreactivity in the neuropil. Similarly, we found that dCed-6, the fly ortholog of C. elegans CED-6, a PTB-domain binding protein that functions genetically downstream of worm CED-1, is expressed in the adult brain in a pattern indistinguishable from Draper. dCed-6 immunoreactivity is strongly reduced in the neuropil through mz0709-Gal4mediated knockdown (with UAS-dCed-6 $6^{R N A i}$ ), and eliminated from the entire brain when $\mathrm{dCed}-6^{\mathrm{RNAi}}$ treatment is performed with the pan-glial driver repo-Gal4. Thus, in the adult brain dCed-6 appears to be expressed exclusively in glia, including cortex glia and ensheathing glia of the neuropil.

Components of the Draper signaling pathway are required specifically in ensheathing glia for glial membrane recruitment to severed ORN axons, and clearance of degenerating axonal debris from the brain. We have shown that knocking down either draper or shark in ensheathing glia is sufficient to block recruitment of glial membranes and Draper to severed axons and clearance of degenerating axonal debris from the CNS. We suspect that dCed-6 is also required in ensheathing glia for a number of reasons. First, dCed-6 and Draper show perfect overlap in expression in the neuropil, and Draper is only expressed in ensheathing glia. Second, ensheathing glia are recruited to degenerating axon injury, and dCed-6, like Draper, is specifically recruited to degenerating maxillary palp ORN axons after maxillary palp ablation. Third, dCed-6 expression is dramatically increased in ensheathing glia surrounding the antennal lobe after antennal ablation, similar to what we have previously found for Draper (MacDonald et al., 2006). Surprisingly, RNAi knock down of dced-6 in ensheathing glia (with $m z 0709-G a l 4$ ) failed to suppress the recruitment of Draper to severed ORN axons or the clearance of axonal debris from the brain. We suspect this is because mz0709-Gal4mediated knockdown of dced-6 is incomplete in ensheathing glia, based on reduced but not eliminated staining in this background. Nevertheless, our observations that dCed-6 is localized with
Draper in immunostains, is required in glia by RNAi knockdown with repo-Gal4, and that a null allele of dced-6 genetically interacts with draper null mutations in axon engulfment, argues strongly for a role for dCed-6 in ensheathing glia.

Moreover, knockdown of draper in ensheathing glia, or suppressing glial engulfing activity by blocking endocytic function with Shibire ${ }^{\text {ts }}$ fully suppresses the recruitment of Draper to severed axons and clearance of degenerating axonal debris from the brain. In contrast, astrocytes do not express Draper (or dCed-6), fail respond morphologically to axon injury, and knockdown of draper in astrocytes has no effect on the recruitment of Draper to severed axons or clearance of axonal debris from the CNS. Such a separation of phagocytic function in neuropil glial cells suggests similarities to the assigned functional roles in mammalian glia, with ensheathing glia as resident phagocytes, engulfing the majority or all axonal debris and astrocytes perhaps playing a less important role in phagocytosis of degenerating axons.

We conclude that ensheathing glia are the phagocytes of the central brain, responsible for engulfing degenerating ORN axons after axotomy. Based on their expression of Draper and dCed-6 we propose that cortex glia play a similar role in the cortex, perhaps engulfing degenerating axons in this tissue, or cell corpses generated during neuronal development or after brain injury. Drosophila astrocytes, in contrast, are in close association with synapse rich regions of the brain and we speculate they likely play an important role in neural circuit and synapse physiology. Our work represents the first functional dissection of glial subtypes in the central brain of adult Drosophila, and lays the foundation for future functional studies of these diverse classes of glia.

\section{References}

Auld VJ, Fetter RD, Broadie K, Goodman CS (1995) Gliotactin, a novel transmembrane protein on peripheral glia, is required to form the bloodnerve barrier in Drosophila. Cell 81:757-767.

Awasaki T, Tatsumi R, Takahashi K, Arai K, Nakanishi Y, Ueda R, Ito K (2006) Essential role of the apoptotic cell engulfment genes draper and ced-6 in programmed axon pruning during Drosophila metamorphosis. Neuron 50:855-867.

Awasaki T, Lai SL, Ito K, Lee T (2008) Organization and postembryonic development of glial cells in the adult central brain of Drosophila. J Neurosci 28:13742-13753.

Bainton RJ, Tsai LT, Schwabe T, DeSalvo M, Gaul U, Heberlein U (2005) moody encodes two GPCRs that regulate cocaine behaviors and bloodbrain barrier permeability in Drosophila. Cell 123:145-156.

Barres BA (2008) The mystery and magic of glia: a perspective on their roles in health and disease. Neuron 60:430-440.

Chaudhry FA, Lehre KP, van Lookeren Campagne M, Ottersen OP, Danbolt NC, Storm-Mathisen J (1995) Glutamate transporters in glial plasma membranes; highly differentiated localizations revealed by quantitative ultrastructural immunocytochemistry. Neuron 15:711-720.

Davalos D, Grutzendler J, Yang G, Kim JV, Zuo Y, Jung S, Littman DR, Dustin ML, Gan WB (2005) ATP mediates rapid microglial response to local brain injury in vivo. Nat Neurosci 8:752-758.

Edenfeld G, Stork T, Klämbt C (2005) Neuron-glia interaction in the insect nervous system. Curr Opin Neurobiol 15:34-39.

Freeman MR, Doherty J (2006) Glial cell biology in Drosophila and vertebrates. Trends Neurosci 29:82-90.

Freeman MR, Delrow J, Kim J, Johnson E, Doe CQ (2003) Unwrapping glial biology. Gcm target genes regulating glial development, diversification, and function. Neuron 38:567-580.

Hidalgo A, Booth GE (2000) Glia dictate pioneer axon trajectories in the Drosophila embryonic CNS. Development 127:393-402.

Hoopfer ED, McLaughlin T, Watts RJ, Schuldiner O, O’Leary DD, Luo L (2006) Wlds protection distinguishes axon degeneration following injury from naturally occurring developmental pruning. Neuron 50:883-895.

Ito K, Urban J, Technau GM (1995) Distribution, classification and devel- 
opment of Drosophila glial cells in the late embryonic and early larval ventral nerve cord. Roux's Arch Dev Biol 204:284-307.

Lee T, Luo L (2001) Mosaic analysis with a repressible cell marker (MARCM) for Drosophila neural development. Trends Neurosci 24:251-254.

Lehre KP, Levy LM, Ottersen OP, Storm-Mathisen J, Danbolt NC (1995) Differential expression of two glial glutamate transporters in the rat brain: quantitative and immunocytochemical observations. J Neurosci 15:1835-1853.

Leiserson WM, Harkins EW, Keshishian H (2000) Fray, a Drosophila serine/ threonine kinase homologous to mammalian PASK, is required for axonal ensheathment. Neuron 28:793-806.

Liu L, Persson JK, Svensson M, Aldskogius H (1998) Glial cell responses, complement, and clusterin in the central nervous system following dorsal root transection. Glia 23:221-238.

Liu QA, Hengartner MO (1998) Candidate adaptor protein CED-6 promotes the engulfment of apoptotic cells in C. elegans. Cell 93:961-972.

Logan MA, Freeman MR (2007) The scoop on the fly brain: glial engulfment functions in the Drosophila. Neuron Glia Biol 3:63-74.

MacDonald JM, Beach MG, Porpiglia E, Sheehan AE, Watts RJ, Freeman MR (2006) The Drosophila cell corpse engulfment receptor Draper mediates glial clearance of severed axons. Neuron 50:869-881.

Matthias K, Kirchhoff F, Seifert G, Hüttmann K, Matyash M, Kettenmann H, Steinhäuser C (2003) Segregated expression of AMPA-type glutamate receptors and glutamate transporters defines distinct astrocyte populations in the mouse hippocampus. J Neurosci 23:1750-1758.

Murray M, Wang SD, Goldberger ME, Levitt P (1990) Modification of as- trocytes in the spinal cord following dorsal root or peripheral nerve lesions. Exp Neurol 110:248-257.

Poeck B, Fischer S, Gunning D, Zipursky SL, Salecker I (2001) Glial cells mediate target layer selection of retinal axons in the developing visual system of Drosophila. Neuron 29:99-113.

Rival T, Soustelle L, Strambi C, Besson MT, Iché M, Birman S (2004) Decreasing glutamate buffering capacity triggers oxidative stress and neuropil degeneration in the Drosophila brain. Curr Biol 14:599-605.

Schwabe T, Bainton RJ, Fetter RD, Heberlein U, Gaul U (2005) GPCR signaling is required for blood-brain barrier formation in Drosophila. Cell 123:133-144

Silies M, Edenfeld G, Engelen D, Stork T, Klämbt C (2007) Development of the peripheral glial cells in Drosophila. Neuron Glia Biol 3:35-43.

Sonnenfeld MJ, Jacobs JR (1995) Macrophages and glia participate in the removal of apoptotic neurons from the Drosophila embryonic nervous system. J Comp Neurol 359:644-652.

Suh J, Jackson FR (2007) Drosophila ebony activity is required in glia for the circadian regulation of locomotor activity. Neuron 55:435-447.

Williams DW, Kondo S, Krzyzanowska A, Hiromi Y, Truman JW (2006) Local caspase activity directs engulfment of dendrites during pruning. Nat Neurosci 9:1234-1236.

Wyss-Coray T, Mucke L (2002) Inflammation in neurodegenerative disease-a double-edged sword. Neuron 35:419-432.

Ziegenfuss JS, Biswas R, Avery MA, Hong K, Sheehan AE, Yeung YG, Stanley ER, Freeman MR (2008) Draper-dependent glial phagocytic activity is mediated by Src and Syk family kinase signalling. Nature 453:935-939. 